\title{
Nongenotoxic effects and a reduction of the DXR-induced genotoxic effects of Helianthus annuus Linné (sunflower) seeds revealed by micronucleus assays in mouse bone marrow
}

\author{
Marcelo Fabiano Gomes Boriollo ${ }^{1,2,3^{*}}$, Luiz Silva Souza ${ }^{1,2}$, Marielly Reis Resende ${ }^{4}$, Thaísla Andrielle da Silva ${ }^{1,2}$,
} Nelma de Mello Silva Oliveira ${ }^{1,4}$, Maria Cristina Costa Resck ${ }^{1,3}$, Carlos Tadeu dos Santos Dias ${ }^{5}$

and João Evangelista Fiorini $i^{1,2}$

\begin{abstract}
Background: This research evaluated the genotoxicity of oil and tincture of $H$. annuus $L$. seeds using the micronucleus assay in bone marrow of mice. The interaction between these preparations and the genotoxic effects of doxorubicin (DXR) was also analysed (antigenotoxicity test).

Methods: Experimental groups were evaluated at 24-48 h post treatment with N-Nitroso-N-ethylurea (positive control - NEU), DXR (chemotherapeutic), $\mathrm{NaCl}$ (negative control), a sunflower tincture (THALS) and two sources of sunflower oils (POHALS and FOHALS). Antigenotoxic assays were carried out using the sunflower tincture and oils separately and in combination with NUE or DXR.
\end{abstract}

Results: For THALS, analysis of the MNPCEs showed no significant differences between treatment doses (2502,000 mg. $\left.\mathrm{Kg}^{-1}\right)$ and $\mathrm{NaCl}$. A significant reduction in MNPCE was observed when THALS $\left(2,000 \mathrm{mg} \cdot \mathrm{Kg}^{-1}\right)$ was administered in combination with DXR $\left(5 \mathrm{mg}^{\mathrm{Kg}}{ }^{-1}\right)$. For POHALS or FOHALS, analysis of the MNPCEs also showed no significant differences between treatment doses $\left(250-2,000 \mathrm{mg} \cdot \mathrm{Kg}^{-1}\right)$ and $\mathrm{NaCl}$. However, the combination DXR + POHALS $\left(2,000 \mathrm{mg} \cdot \mathrm{Kg}^{-1}\right)$ or DXR + FOHALS $\left(2,000 \mathrm{mg} \cdot \mathrm{Kg}^{-1}\right)$ not contributed to the MNPCEs reduction.

Conclusions: This research suggests absence of genotoxicity of THALS, dose-, time- and sex-independent, and its combination with DXR can reduce the genotoxic effects of DXR. POHALS and FOHALS also showed absence of genotoxicity, but their association with DXR showed no antigenotoxic effects.

Keywords: Bone marrow, Helianthus annuus L. (sunflower), Micronucleus assay, Rodents, Tincture, Oil

\section{Background}

The cultivated sunflower (Helianthus annuus L.) is one of 67 species in the genus Helianthus. It is a dicotyledonous plant and a member of the Compositae (Asteraceae) family, having a typical composite flower [1]. The composition of the seed is markedly affected by

\footnotetext{
* Correspondence: marcelo.boriollo@unifenas.br

'Laboratório de Farmacogenômica e Biologia Molecular, Faculdade de Ciências Médicas \& Centro de Pesquisa e Pós-graduação, Universidade José do Rosário Vellano (UNIFENAS), Campus Universitário, Rod. MG 179, Km 0, Alfenas, MG CEP: 37130-000, Brasil

${ }^{2}$ Centro de Pesquisa e Pós-graduação em Ciência Animal, Área de Patologia e Farmacologia Animal, Universidade José do Rosário Vellano (UNIFENAS), Alfenas, Minas Gerais, Brasil

Full list of author information is available at the end of the article
}

the sunflower variety [2,3]. Nevertheless, the composition ranges of sunflower dehulled seeds (on a percentage dry weight basis) is as follows [4]: protein ${ }^{20.4-40.0 \%}$; peptides, amino acids and other non-protein nitrogen ${ }^{1-13 \%}$; carbohydrates ${ }^{4-10 \%}$; lipids ${ }^{47-65 \%}$; fatty acids (palmitic acid ${ }^{5-7 \%}$, atearic ${ }^{2-6 \%}$; arachidic acid ${ }^{0.0-0.3 \%}$, oleic acid ${ }^{15-37 \%}$; linoleic acid ${ }^{51-73 \%}$, and linolenic acid ${ }^{<0.3 \%}$ ); tocopherol ${ }^{0.07 \%}$; carotenoids ${ }^{0.01-0.02 \%}$; vitamin B1 ${ }^{0.002 \%}$; chlorogenic acid (CGA) ${ }^{0.5-2.4 \%}$; quinic acid (QA) ${ }^{0.12-0.25 \%}$; caffeic acid (CA) ${ }^{0.05-0.29 \%}$; total minerals ${ }^{3-4 \%}$; potassium ${ }^{0.67-0.75 \%}$; phosphorus ${ }^{0.60-0.94 \%}$; sulphur ${ }^{0.26-0.32 \%}$; magnesium ${ }^{0.35-0.41 \%}$; calcium $^{0.08-0.10 \%}$; and sodium ${ }^{0.02 \%}$. 
Tocopherols are excellent natural antioxidants that protect oils against oxidative rancidity. The $\alpha$ form has the highest biological vitamin E activity, and the $\gamma$ form has been reported to have the highest antioxidant activity [5]. The sterols found in sunflower oils include $\beta$-sitosterol, stigmasterol, campesterol, $\delta$-5-avenasterol, and $\delta$-7-stigmasterol $[6,7]$. Plant sterols are only minimally absorbed by humans, and their ingestion appears to inhibit intestinal cholesterol and bile acid absorption [8]. Most trace metals in refined, bleached and deodorized sunflower seed oil are removed during processing. It is particularly important that copper and iron be removed because these metals greatly reduce the oxidative stability of the oil [9]. Other metals, such as lead and cadmium, are of particular concern due to their toxicity and their supposed link to coronary heart disease and hypertension [10].

In drug development, the genotoxicity assays represent a considerable effort, as most pharmaceutical organizations evaluate a new therapeutic agent based on in vitro and in vivo data genotoxic [11]. In this context, tests to evaluate the genotoxic activity of the plants used by the population as well as their isolated compounds are necessary and important for establishing control measures in widespread use. Furthermore, it is necessary to clarify the mechanisms and conditions that mediate the proposed biological effect before plants are considered as therapeutic agents [12]. As far as genotoxicity studies are concerned, the in vivo micronucleus (MN) assay in rodent bone marrow plays a crucial role in the test battery aimed at identifying hazardous mutagens [13]; this assay is especially suited to assessing genotoxic hazards because it allows consideration of multiple factors, such as in vivo metabolism, pharmacokinetics and DNA repair processes, even though these processes vary among species, among tissues and among genetic endpoints [14-17]. In addition, understanding the genotoxic effects induced by phytotherapeutics and foods employing the mammalian in vivo $\mathrm{MN}$ assay has been the goal of several researchers groups [18-20].

In order to contribute to the information on the genotoxic potential of herbal extracts and food, the present study evaluated the genotoxic effects of two sources of oil and tincture of $H$. annuus L. (sunflower) seeds using in vivo micronucleus assays in mouse bone marrow. The effect of the maximum permissible concentration of $H$. annuus L. (oils and tincture) on the doxorubicin (DXR)-induced genotoxic effects in mice bone marrow was also studied (i.e., antigenotoxicity assay).

\section{Methods}

\section{Phytotherapeutics}

Tincture and oil of sunflower seeds were purchased commercially and stored according to the manufacturer's recommendations [tincture of $H$. annuus L. seeds (THALS) - Yod Comércio de Produtos Naturais Ltda., cat. \# 544606, Campinas, SP, Brazil; pharmaceutical oil of $H$. annuus L. seeds (POHALS) - Farmácia de Manipulação Alfenense Ltda., Alfenas, MG, Brazil; food oil of $H$. annuus L. seeds (FOHALS) - Agricultural Cargill S.A., Mairinque, SP, Brazil]. Aliquots $(1.5 \mathrm{~L})$ of this tincture were submitted to solvent removal proceedings by rotary evaporation (40 rpm) (Rotavapor Model R-215) coupled in bath heating systems ${ }^{50-60^{\circ} \mathrm{C}}$ (Bath Heating model B-491), vacuum pump $500 \mathrm{mmHg}$ (Vacuum Pump V-700 with Automatic Vacuum Controller V-855), recirculator (Recirculator Chiller F-100) and evaporation bottle (Büchi Labortechnik AG, Switzerland). The final product was transferred to a reaction bottle $1 \mathrm{~L}\left(\mathrm{SCHOTT}^{\circ}\right.$ DURAN ${ }^{\circ}$ ) and kept at $-20^{\circ} \mathrm{C}$ for 24 hours in order to evaluate the freezing of the final product and the efficacy of the solvent evaporation process [21]. Then, aliquots $(40 \mathrm{~mL})$ of this final product was transferred into glass vials penicillin type $(50 \mathrm{~mL})$ and lyophilized (Lyophilizer model Alpha 1-2 LDPlus, Martin Christ Gefriertrocknungsanlagen $\mathrm{GmbH}^{\odot}$, Germany) and their dry mass were measured (Electronic Analytical Balance AUW-220D, Shimadzu Corp., Kyoto, Japan). The lyophilized final product was prepared in aqueous solvent (150 mM NaCl in water type 1) at concentrations of $2 \times$, sterilized by filtration (Millipore Corporation, hydrophilic Durapore PVDF, $0.22 \mu \mathrm{m}, \varnothing 47 \mathrm{~mm}$, cat. \# GVWP $04700)$, and stored in sterile polypropylene tubes $(50 \mathrm{~mL})$ at $-70^{\circ} \mathrm{C}$ until moment of use.

\section{System - test in vivo}

Healthy, heterogeneous, young adult male and female Swiss albinus (Unib: SW) mice (between 7 and 12 weeks pubescent period), with a body weight between $30 \mathrm{~g}$ and $40 \mathrm{~g}$ (i.e., the variation weight between the animals, for each sex, should not exceed the $\pm 20 \%$ of medium mass) were provided by CEMIB (Centro Multidisciplinar para Investigação Biológica na Área da Ciência em Animais de Laboratório - UNICAMP; http://www.cemib.unicamp.br/), and erythrocytes from the bone marrow of these mice were used in the micronucleus assay $[14,17,22]$. The animals were kept in groups of the same sex, in polypropylene boxes, in an air-conditioned environment to $22^{\circ} \mathrm{C} \pm 3^{\circ} \mathrm{C}$, with relative air humidity of $50 \% \pm 20 \%$, and with $12-$ hour day-night cycles (i.e., $12 \mathrm{~h}$ light and $12 \mathrm{~h}$ dark). These were fed with Purina Labina commercial rations (Nestlé Purina Pet Care Company) and water ad libitum, and acclimated to laboratory conditions for 7 days (a trial period) before the execution of the experiment. At the end of the trial period, each animal was weighed and, according to the weight, received $2 \mathrm{~mL} / 100 \mathrm{~g}$ body weight of the indicated liquid (negative control, positive control, chemotherapeutic and phytotherapeutic). After 
the experimental treatment, the animals were euthanized by $\mathrm{CO}_{2}$ asphyxiation in adapted acrylic chambers [14]. This research was approved by Committee of Ethics in Research Involving Animals of UNIFENAS (CEPEAU Protocol No. 04A/2008).

\section{Experimental groups}

Groups of animals (consisting of 3 males and 3 females each) were treated using a single dosing regimen administered by gavage (phytotherapeutic and negative control) or intraperitoneally (chemotherapeutic and positive control) and two euthanasia times ( 24 and $48 \mathrm{~h}$ ), based on a regulatory recommendation regarding the in vivo micronucleus assay [14,17]:

- Control groups: $150 \mathrm{mM} \mathrm{NaCl}$ (negative control), $50 \mathrm{mg} \cdot \mathrm{Kg}^{-1}$ of N-Nitroso-N-ethylurea (positive control: NEU, Sigma N8509, CAS no. 759-73-9) and $5 \mathrm{mg} \cdot \mathrm{Kg}^{-1}$ of doxorubicin hydrochloride [20] (chemotherapeutic: DXR, Eurofarma Laboratórios Ltda., CAS no. 23214-92-8).

- Genotoxicity test (phytotherapeutics): THALS (250-2,000 mg. $\left.\mathrm{Kg}^{-1}\right)$, POHALS (250-2,000 mg. $\mathrm{Kg}^{-1}$ ) and FOHALS (250-2,000 mg. $\mathrm{Kg}^{-1}$ ). The maximum tolerated dose (MTD) was defined as $(i)$ the highest dose that can be administered without inducing lethality or excessive toxicity during the study causing moribund euthanasia, or (ii) a dose that produces some indication of toxicity of the bone marrow (e.g. a reduction in the proportion of immature erythrocytes among total erythrocytes in the bone marrow), or (iii) 2,000 mg. $\mathrm{Kg}^{-1}[14,17]$.

- Antigenotoxicity test 1 (phytotherapeutics +

chemotherapeutic) [20]: THALS (2,000 mg. $\left.\mathrm{Kg}^{-1}\right)+$ DXR (5 mg. $\left.\mathrm{Kg}^{-1}\right)$, FOHALS (2,000 mg. $\left.\mathrm{Kg}^{-1}\right)+$ DXR $\left(5 \mathrm{mg} \cdot \mathrm{Kg}^{-1}\right)$ and FOHALS (2,000 mg. $\left.\mathrm{Kg}^{-1}\right)+$ DXR $\left(5 \mathrm{mg} \cdot \mathrm{Kg}^{-1}\right)$.

- Antigenotoxicity test 2 (phytotherapeutics + positive control): THALS (2,000 mg. $\left.\mathrm{Kg}^{-1}\right)+\mathrm{NEU}\left(50 \mathrm{mg} \cdot \mathrm{Kg}^{-1}\right)$, POHALS (2,000 mg. Kg $\left.{ }^{-1}\right)+\mathrm{NEU}\left(50 \mathrm{mg} \cdot \mathrm{Kg}^{-1}\right)$ and POHALS (2,000 mg. Kg $\left.{ }^{-1}\right)+\mathrm{NEU}\left(50 \mathrm{mg} \cdot \mathrm{Kg}^{-1}\right)$.

\section{Processing the bone marrow and cell analysis}

Shortly after euthanasia, the femora were surgically and aseptically removed, and the animals appropriately discarded. Each femur was sectioned at the proximal end and the contents of the spinal canal were washed with $1.5 \mathrm{~mL}$ of $150 \mathrm{mM} \mathrm{NaCl}$ solution and transferred to a $15 \mathrm{~mL}$ centrifuge tube $[14,17,23]$. This material was resuspended with a Pasteur pipette to ensure a random distribution of bone marrow cells. The suspension was then centrifuged at 1,000 rpm (Centrifuga de Bancada Microprocessada, Mod. NT 810, Nova Técnica Ind. e Com. de Equip. para Laboratório Ltda., Piracicaba, SP,
Brazil) for 5 minutes. The supernatant was discarded and the resulting sediment was resuspended in $500 \mu \mathrm{L}$ of $150 \mathrm{mM} \mathrm{NaCI}$ solution added 4\% formaldehyde. The slides were prepared by smearing ( 2 slides per animal), dried at room temperature for $24 \mathrm{~h}$ and stained with Leishman's eosin methylene blue dye [pure dye for $3 \mathrm{~min}$, followed by diluted dye in water type 1 (1:6) for $15 \mathrm{~min}$ ] to differentiate polychromatic erythrocyte (PCE) from normochromatic erythrocyte (NCE).

Polychromatic erythrocytes (PCEs) were observed at a magnification of 1000× using optical microscopy (Nikon Eclipse E-200), counted (at least 2000 polychromatic erythrocytes anucleated per animal were scored for the incidence of micronucleated polychromatic erythrocytes) with the aid of a digital cell counter (Contador Diferencial CCS02, Kacil Indústria e Comércio Ltda., PE, Brasil Contador Diferencial CCS02, Kacil Indústria e Comércio Ltda., PE, Brazil) and photographed using an 8.1 Megapixel Digital Camera (DC FWL 150). The number of PCEs and NCEs, the number and frequency of micronucleated polychromatic erythrocytes (MNPCEs) were reported. In order to evaluate bone-marrow toxicity, the ratio of PCE to NCE was also observed [14,17]. This PCE/NCE ratio is an indicator of the acceleration or inhibition of erythropoiesis and it has been reported to vary with scoring time. A continuous decline in the PCE/NCE ratio may be due to the inhibition of cell division, the killing of erythroblasts, the removal of damaged cells, or dilution of the existing cell pool with newly formed cells [20].

\section{Statistical analysis}

The data obtained in the micronucleus assay were submitted to one-way analysis of variance (ANOVA), using a factorial scheme of $10 \times 2 \times 2$ (treatment $\times$ sex $\times$ euthanasia time), and medium comparison with Tukey's test $(\alpha=0.05)$ using SAS version 9.2 computer software.

\section{Results and discussion}

$H$. annuus L. has been considered an important source of natural oil for centuries and has been used as a preventive medicine against diuresis, diarrhoea, and various inflammatory diseases [24], and has also been used for the relief of asthmatic symptoms [25], gastric protection $[26,27]$, its healing properties [28], anti-inflammatory action [29] and antimicrobial properties [26,28]. However, studies aimed at understanding the genotoxic and mutagenic effects of $H$. annuus L. were subject of comparatively little research $[19,30]$, which drove us to evaluate the harmful genotoxic and antigenotoxic properties (i.e., clastogenicity and/or aneugenicity) of oil and tincture of $H$. annuus L. seeds using the MN assay in vivo.

The numbers and frequencies of MNPCEs and the $\mathrm{PCE} / \mathrm{NCE}$ ratio in the bone marrow of mice were analyzed 
statistically for each one of the animal groups treated with only tincture (THALS) or oils (POHALS or FOHALS) of sunflower seeds - genotoxic assays - and for each one of the groups treated with phytotherapeutics and chemotherapeutic agent DXR (THALS + DXR, POHALS + DXR or FOHALS + DXR) - antigenotoxic assays -, as well as control groups.

For animal groups treated with THALS, analysis of the MNPCEs showed no significant differences $(p<0.05)$ between all the treatment doses (250-2,000 mg. $\mathrm{Kg}^{-1}$ ) and negative control $(\mathrm{NaCl})$. These results suggest absence of genotoxicity of THALS, regardless of the dose of phytotherapic administration (250-2,000 mg. $\mathrm{Kg}^{-1}$ ), the treatment time ( 24 and $48 \mathrm{~h}$ ) or the sex of the animal (male and female). Treatment of mice with $5 \mathrm{mg} \cdot \mathrm{Kg}^{-1}$ DXR significantly induced MNPCE at 24 and $48 \mathrm{~h}$ post treatment and for both sexes, whose MNPCE frequencies were significantly above $(p<0.05)$ those observed in the positive NEU control $\left(50 \mathrm{mg} \cdot \mathrm{Kg}^{-1}\right)$. However, the reduction in MNPCE $(p<0.05)$ was observed when THALS $\left(2,000 \mathrm{mg} \cdot \mathrm{Kg}^{-1}\right)$ is administered in combination with the chemotherapy agent DXR $\left(5 \mathrm{mg} \cdot \mathrm{Kg}^{-1}\right)$, suggesting antigenotoxic effects (anticlastogeny and/or antianeugeny). Therefore, THALS provides a partial protection against the genotoxic effects induced by DXR in the bone marrow of mice, regardless of the treatment time ( 24 and $48 \mathrm{~h}$ ) or the sex of the animal, although the genotoxic effect observed in this treatment combination has is similar (i.e., numbers and frequencies of MNPCEs) to that observed in NEUtreated animals. The analysis obtained from the PCE/NCE ratio showed no significant differences $(p<0.05)$ between all doses of THALS (250-2,000 mg. $\left.\mathrm{Kg}^{-1}\right)$, THALS $\left(2,000 \mathrm{mg} \cdot \mathrm{Kg}^{-1}\right)+$ DXR $\left(5 \mathrm{mg} \cdot \mathrm{Kg}^{-1}\right)$ and negative controls. These results suggest that there is not systemic toxicity of THALS and/or DXR under the MN assay conditions, regardless of the phytotherapeutic doses and times, but sex-dependent (Table 1).

For animal groups treated with POHALS (Table 2) or FOHALS (Table 3), analysis of the MNPCEs showed no significant differences $(p<0.05)$ between all the treatment doses (250-2,000 mg. $\left.\mathrm{Kg}^{-1}\right)$ and negative control $(\mathrm{NaCl})$. These results suggest absence of genotoxicity for both sources of sunflower oil (pharmaceutical and food), regardless of the dose of oil administration $\left(250-2,000 \mathrm{mg} \cdot \mathrm{Kg}^{-1}\right)$ or treatment time (24 and $48 \mathrm{~h}$ ), but it was sex-dependent. Treatment of mice with DXR (5 mg. $\left.\mathrm{Kg}^{-1}\right)+$ POHALS $\left(2,000 \mathrm{mg} \cdot \mathrm{Kg}^{-1}\right)$ or DXR (5 mg. $\left.\mathrm{Kg}^{-1}\right)+$ FOHALS $\left(2,000 \mathrm{mg} \cdot \mathrm{Kg}^{-1}\right)$ not contribute to the MNPCEs reduction at 24 and $48 \mathrm{~h}$ post treatment and for both sexes, suggesting that both sources of sunflower oil not decrease the DXR-induced genotoxic effects and therefore they do not have antigenotoxic effects (anticlastogeny and/or antianeugeny). The analysis obtained from the PCE/NCE ratio showed no significant differences $(p<0.05)$ between all doses of POHALS (250-2,000 mg. $\mathrm{Kg}^{-1}$ ) and negative controls, time-dependent and sex-independent. For FOHALS, the PCE/NCE ratio showed significant differences $(p<0.05)$ only in the highest dose $\left(2,000 \mathrm{mg} \cdot \mathrm{Kg}^{-1}\right)$ tested, timeindependent and sex-dependent. These results suggest that the systemic toxicity of sunflower oil can be dependent on its source and its highest dose used. In addition, treatments with DXR (5 mg. $\left.\mathrm{Kg}^{-1}\right)+$ POHALS $\left(2,000 \mathrm{mg} \cdot \mathrm{Kg}^{-1}\right)$ or DXR (5 mg. $\left.\mathrm{Kg}^{-1}\right)+$ FOHALS (2,000 mg. $\left.\mathrm{Kg}^{-1}\right)$ significantly decrease the PCE/NCE ratio in mouse bone marrow. These results suggests that the association sunflower oil and chemotherapeutic agent DXR can potentize the systemic toxicity, regardless of the sex (only POHALS) and time (only FOHALS).

For the first time, this research has provided information on the genotoxic and antigenotoxic effects of THALS. However, genotoxic studies of sunflower oil and oil sunflower ozonized (at a dose limit of $2 \mathrm{~g} \cdot \mathrm{kg}^{-1} \cdot \mathrm{d}^{-1}$, based on evidence of toxicity from subchronic studies via intragastric administration of the product) were previously carried out using the MN assay in the bone marrow of mice using male and female Cenp: NMRI mice [31]. In this study, the treatment with sunflower oil did not cause cytotoxic damage to erithrocytes, as reported in the analyses of the PCE/NCE ratio, which corroborate with our findings from the pharmaceutical oil and partially with food oil. Likewise, that research proposes the hypothesis that no clastogenic effect occurs in the bone marrow of animals treated with the sunflower oil under experimental conditions [31].

Other studies have investigated the suitability of different vegetable oils for the human diet, reporting reductions in genotoxicity and cancer potentiation by sesame oil [32] sunflower oil [33], perilla and palm oil [34], olive, sunflower, peanut, corn, and soy oils [35], flax seed oil [36], and coconut oil [37], among others. The possible role of fatty acids, a main component of vegetable oils, in modulating genotoxicity and carcinogenicity has also been studied. The genotoxic activity of vegetable oils [seed oils of sesame, sunflower, wheat germ, flax, and soy oil, and both first-class extra-virgin and low-grade (refined) olive oil] consumed by humans were also tested in a Drosophila somatic mutation and recombination test (the Drosophila melanogaster SMART assay) [30]. Flax oil produced the strongest response, while sesame, wheat germ, and soy oil showed some genotoxic activity. Sunflower oil and the low-grade olive oil gave inconclusive results or negative biological diagnoses, possibly due to lower concentrations of PUFAs, even as refined products, and extra-virgin olive oil was clearly not genotoxic. It has been argued that the genotoxicity of an oil is most likely due to the fatty acid composition of the oil, which after peroxidation can form 
Table 1 The incidence of MNPCEs and PCE/NCE ratio in bone marrow of male and female Swiss albinus mice after testing for $24 \mathrm{~h}$ and $48 \mathrm{~h}$

\begin{tabular}{|c|c|c|c|c|c|c|c|c|c|c|}
\hline \multirow[t]{2}{*}{ Treatment } & \multicolumn{2}{|c|}{ Number of PCEs analyzed } & \multicolumn{4}{|c|}{ PCEMNs } & \multicolumn{2}{|c|}{$\mathrm{PCE} /(\mathrm{PCE}+\mathrm{NCE})$} & \multicolumn{2}{|l|}{$\operatorname{NCE}(n)$} \\
\hline & $24 \mathrm{~h}$ & $48 \mathrm{~h}$ & $\begin{array}{l}24 \mathrm{~h} \\
(n)^{\mathrm{A}}\end{array}$ & $\begin{array}{l}48 \mathrm{~h} \\
(n)^{\mathrm{A}}\end{array}$ & $\begin{array}{l}24 \mathrm{~h} \\
(\%){ }^{A^{\prime}}\end{array}$ & $\begin{array}{l}48 \mathrm{~h} \\
(\%)^{A^{\prime}}\end{array}$ & $24 h^{A^{\prime \prime}}$ & $48 h^{B^{\prime \prime}}$ & $24 \mathrm{~h}$ & $48 \mathrm{~h}$ \\
\hline \multicolumn{11}{|c|}{$150 \mathrm{mM} \mathrm{NaCl}$} \\
\hline$q_{1}$ & 2095 & 2097 & 7 & 10 & 0.33 & 0.48 & 1.00 & 1.00 & 5 & 3 \\
\hline $\mathrm{q}_{2}$ & 2094 & 2095 & 9 & 10 & 0.43 & 0.48 & 1.00 & 1.00 & 6 & 5 \\
\hline $\mathrm{P}_{3}$ & 2087 & 2089 & 11 & 8 & 0.53 & 0.38 & 0.99 & 0.99 & 13 & 11 \\
\hline$\Sigma q$ & $\Sigma 6276$ & $\Sigma 6281$ & $\sum 27$ & $\Sigma 28$ & $0.43 \pm 0.10$ & $0.45 \pm 0.05$ & $1.00 \pm 0.00$ & $1.00 \pm 0.00$ & $\sum 24$ & $\Sigma 19$ \\
\hline$\widehat{\partial}_{1}$ & 2095 & 2088 & 9 & 13 & 0.43 & 0.62 & 1.00 & 0.99 & 5 & 12 \\
\hline$\hat{\mathrm{O}}_{2}$ & 2055 & 2088 & 12 & 11 & 0.58 & 0.53 & 0.98 & 0.99 & 45 & 12 \\
\hline$\hat{o}_{3}$ & 2058 & 2084 & 7 & 11 & 0.34 & 0.53 & 0.98 & 0.99 & 42 & 16 \\
\hline$\Sigma \hat{O}$ & $\Sigma 6208$ & $\Sigma 6260$ & $\sum 28$ & $\Sigma 35$ & $0.45 \pm 0.12$ & $0.56 \pm 0.06$ & $0.99 \pm 0.01$ & $0.99 \pm 0.00$ & $\sum 92$ & $\sum 40$ \\
\hline$\Sigma \hat{o}$ and $q$ & $\sum 12484$ & $\Sigma 12541$ & $\sum 55^{\mathrm{A}}$ & $\sum 63^{\mathrm{A}}$ & $0.44 \pm 0.08^{A^{\prime}}$ & $0.50 \pm 0.06^{\mathrm{A}^{\prime}}$ & $0.99 \pm 0.01 \mathrm{~A}^{\prime \prime}$ & $1.00 \pm 0.00 \mathrm{~A}^{\prime \prime}$ & $\Sigma 116$ & $\Sigma 59$ \\
\hline \multicolumn{11}{|c|}{ N-Nitroso-N-ethylurea - NEU (50 mg. $\left.\mathrm{Kg}^{-1}\right)$} \\
\hline$q_{1}$ & 2148 & 2075 & 38 & 36 & 1.77 & 1.73 & 0.49 & 0.65 & 2252 & 1125 \\
\hline $\mathrm{O}_{2}$ & 1884 & 2032 & 32 & 34 & 1.70 & 1.67 & 0.54 & 0.81 & 1616 & 468 \\
\hline $\mathrm{q}_{3}$ & 2002 & 1948 & 15 & 31 & 0.75 & 1.59 & 0.61 & 0.93 & 1298 & 152 \\
\hline$\Sigma q$ & $\Sigma 6034$ & $\Sigma 6055$ & $\sum 85$ & $\sum 101$ & $1.41 \pm 0.57$ & $1.67 \pm 0.07$ & $0.54 \pm 0.06$ & $0.80 \pm 0.14$ & $\sum 5166$ & $\sum 1745$ \\
\hline$\sigma_{1}$ & 2025 & 1999 & 64 & 31 & 3.16 & 1.55 & 0.41 & 0.36 & 2875 & 3501 \\
\hline$\widehat{\partial}_{2}$ & 2028 & 1916 & 105 & 40 & 5.18 & 2.09 & 0.51 & 0.55 & 1972 & 1584 \\
\hline$\hat{\partial}_{3}$ & 2004 & 2069 & 25 & 38 & 1.25 & 1.84 & 0.67 & 0.65 & 996 & 1131 \\
\hline$\sum 0^{\pi}$ & $\sum 6057$ & $\sum 5984$ & $\sum 194$ & $\sum 109$ & $3.20 \pm 1.97$ & $1.83 \pm 0.27$ & $0.53 \pm 0.13$ & $0.52 \pm 0.14$ & $\Sigma 5843$ & $\sum 6216$ \\
\hline$\Sigma \hat{o}$ and $q$ & $\Sigma 12091$ & $\Sigma 12039$ & $\sum 279^{B}$ & $\Sigma 210^{B}$ & $2.30 \pm 1.66^{B^{\prime}}$ & $1.75 \pm 0.18^{B^{\prime}}$ & $0.54 \pm 0.06^{B^{\prime \prime}}$ & $0.66 \pm 0.16^{\mathrm{B}^{\prime \prime}}$ & $\Sigma 11009$ & $\Sigma 7961$ \\
\hline \multicolumn{11}{|c|}{ Doxorubicin hydrochloride - DXR (5 mg. $\left.\mathrm{Kg}^{-1}\right)$} \\
\hline$q_{1}$ & 2091 & 2017 & 49 & 36 & 2.34 & 1.78 & 0.72 & 0.96 & 809 & 83 \\
\hline $\mathrm{O}_{2}$ & 2106 & 2077 & 73 & 63 & 3.47 & 3.03 & 0.98 & 0.99 & 44 & 23 \\
\hline$q_{3}$ & 2056 & 2092 & 57 & 50 & 2.77 & 2.39 & 0.84 & 0.95 & 394 & 108 \\
\hline$\Sigma q$ & $\sum 6253$ & $\sum 6186$ & $\sum 179$ & $\sum 149$ & $2.86 \pm 0.57$ & $2.40 \pm 0.62$ & $0.85 \pm 0.13$ & $0.97 \pm 0.02$ & $\sum 1247$ & $\sum 214$ \\
\hline$\widehat{\partial}_{1}$ & 2067 & 2086 & 53 & 61 & 2.56 & 2.92 & 0.98 & 0.95 & 33 & 114 \\
\hline$\hat{\mathrm{o}}_{2}$ & 2063 & 2042 & 56 & 70 & 2.71 & 3.43 & 0.98 & 0.97 & 37 & 58 \\
\hline$\hat{O}_{3}$ & 2082 & 2075 & 46 & 50 & 2.21 & 2.41 & 0.99 & 0.99 & 18 & 25 \\
\hline$\sum \sigma^{\lambda}$ & $\Sigma 6212$ & $\sum 6203$ & $\sum 155$ & $\sum 181$ & $2.50 \pm 0.26$ & $2.92 \pm 0.51$ & $0.99 \pm 0.00$ & $0.97 \pm 0.02$ & $\sum 88$ & $\sum 197$ \\
\hline$\Sigma \widehat{o}$ and $q$ & $\sum 12465$ & $\Sigma 12389$ & $\Sigma 334^{c}$ & $330^{c}$ & $2.68 \pm 0.42^{C^{\prime}}$ & $2.66 \pm 0.43^{C^{\prime}}$ & $0.92 \pm 0.07 \mathrm{~A}^{\prime \prime}$ & $0.97 \pm 0.01 \mathrm{~A}^{\mathrm{A}}$ & $\Sigma 1335$ & $\sum 411$ \\
\hline \multicolumn{11}{|c|}{ THALS - Tincture of H. annuus L. seeds (250 mg. $\mathrm{Kg}^{-1}$ ) } \\
\hline$q_{1}$ & 2097 & 2192 & 8 & 7 & 0.38 & 0.32 & 0.99 & 0.99 & 14 & 12 \\
\hline $\mathrm{q}_{2}$ & 2105 & 2057 & 7 & 12 & 0.33 & 0.58 & 0.99 & 0.99 & 31 & 28 \\
\hline$q_{3}$ & 2181 & 2092 & 12 & 12 & 0.55 & 0.57 & 0.99 & 1.00 & 19 & 8 \\
\hline$\Sigma q^{\mathrm{AA}} \mathrm{A}$ & $\Sigma 6383$ & $\Sigma 6341$ & $\sum 27$ & $\Sigma 31$ & $0.42 \pm 0.11$ & $0.49 \pm 0.15$ & $0.99 \pm 0.00$ & $0.99 \pm 0.01$ & $\Sigma 64$ & $\sum 48$ \\
\hline$\hat{o}_{1}$ & 2041 & 2070 & 7 & 7 & 0.34 & 0.34 & 0.99 & 1.00 & 27 & 10 \\
\hline$\hat{O}_{2}$ & 2050 & 2062 & 9 & 6 & 0.44 & 0.29 & 0.99 & 0.99 & 17 & 19 \\
\hline$\hat{o}_{3}$ & 2055 & 2065 & 10 & 12 & 0.49 & 0.58 & 0.99 & 0.99 & 12 & 15 \\
\hline$\Sigma \overbrace{}^{A A}$ & $\Sigma 6146$ & $\sum 6197$ & $\Sigma 26$ & $\Sigma 25$ & $0.42 \pm 0.07$ & $0.40 \pm 0.16$ & $0.99 \pm 0.00$ & $0.99 \pm 0.00$ & $\Sigma 56$ & $\sum 44$ \\
\hline$\Sigma \widehat{o}$ and $q$ & $\Sigma 12529$ & $\Sigma 12538$ & $\sum 53^{A}$ & $\sum 56^{\mathrm{A}}$ & $0.42 \pm 0.09^{A^{\prime}}$ & $0.45 \pm 0.14^{\mathrm{A}^{\prime}}$ & $0.99 \pm 0.00 \mathrm{~A}^{\prime \prime}$ & $0.99 \pm 0.00 \mathrm{~A}^{\prime \prime}$ & $\Sigma 120$ & $\Sigma 92$ \\
\hline
\end{tabular}


Table 1 The incidence of MNPCEs and PCE/NCE ratio in bone marrow of male and female Swiss albinus mice after testing for $24 \mathrm{~h}$ and $\mathbf{4 8} \mathrm{h}$ (Continued)

\begin{tabular}{|c|c|c|c|c|c|c|c|c|c|c|}
\hline \multicolumn{11}{|c|}{ THALS - Tincture of H. annuus L. seeds (500 mg. $\left.\mathrm{Kg}^{-1}\right)$} \\
\hline$q_{1}$ & 2086 & 2146 & 12 & 16 & 0.58 & 0.75 & 0.99 & 0.99 & 17 & 22 \\
\hline $\mathrm{q}_{2}$ & 2078 & 2060 & 12 & 13 & 0.58 & 0.63 & 0.99 & 0.99 & 25 & 11 \\
\hline$q_{3}$ & 2072 & 2046 & 13 & 11 & 0.63 & 0.54 & 0.99 & 0.99 & 30 & 26 \\
\hline$\sum q^{\mathrm{AA}}$ & $\sum 6236$ & $\Sigma 6252$ & $\sum 37$ & $\sum 40$ & $0.59 \pm 0.03$ & $0.64 \pm 0.10$ & $0.99 \pm 0.00$ & $0.99 \pm 0.00$ & $\Sigma 72$ & $\Sigma 59$ \\
\hline$\hat{o}_{1}$ & 2071 & 2075 & 17 & 8 & 0.82 & 0.39 & 0.98 & 0.99 & 32 & 13 \\
\hline$\hat{\partial}_{2}$ & 2074 & 2081 & 12 & 11 & 0.58 & 0.53 & 0.99 & 0.99 & 29 & 20 \\
\hline$\hat{o}_{3}$ & 2072 & 2067 & 11 & 10 & 0.53 & 0.48 & 0.99 & 0.99 & 22 & 11 \\
\hline$\sum \delta^{A A}$ & $\sum 6217$ & $\Sigma 6223$ & $\sum 40$ & $\sum 29$ & $0.64 \pm 0.16$ & $0.47 \pm 0.07$ & $0.99 \pm 0.00$ & $0.99 \pm 0.00$ & $\sum 83$ & $\sum 44$ \\
\hline$\Sigma \hat{o}$ and $q$ & $\Sigma 12453$ & $\sum 12475$ & $\sum 77^{\mathrm{A}}$ & $\sum 69^{A}$ & $0.62 \pm 0.10^{A^{\prime}}$ & $0.55 \pm 0.12^{\mathrm{A}^{\prime}}$ & $0.99 \pm 0.00 \mathrm{~A}^{\prime \prime}$ & $0.99 \pm 0.00 \mathrm{~A}^{\prime \prime}$ & $\Sigma 155$ & $\sum 103$ \\
\hline \multicolumn{11}{|c|}{ THALS - Tincture of H. annuus L. seeds $\left(1,000 \mathrm{mg} \cdot \mathrm{Kg}^{-1}\right)$} \\
\hline$q_{1}$ & 2083 & 2165 & 13 & 17 & 0.62 & 0.79 & 0.98 & 0.99 & 35 & 17 \\
\hline $\mathrm{q}_{2}$ & 2075 & 2076 & 12 & 14 & 0.58 & 0.67 & 0.99 & 0.99 & 24 & 25 \\
\hline q3 $_{3}$ & 2070 & 2061 & 13 & 14 & 0.63 & 0.68 & 0.98 & 0.98 & 49 & 34 \\
\hline$\sum q^{A A}$ & $\sum 6228$ & $\Sigma 6302$ & $\sum 38$ & $\sum 45$ & $0.61 \pm 0.03$ & $0.71 \pm 0.06$ & $0.98 \pm 0.01$ & $0.99 \pm 0.00$ & $\sum 108$ & $\sum 76$ \\
\hline$\hat{o}_{1}$ & 2079 & 2084 & 18 & 10 & 0.87 & 0.48 & 0.98 & 0.99 & 32 & 31 \\
\hline$\hat{O}_{2}$ & 2092 & 2095 & 13 & 11 & 0.62 & 0.53 & 0.99 & 0.99 & 30 & 21 \\
\hline$\hat{\partial}_{3}$ & 2073 & 2077 & 11 & 8 & 0.53 & 0.39 & 0.98 & 0.98 & 32 & 40 \\
\hline$\sum \sigma^{A A}$ & $\Sigma 6244$ & $\Sigma 6256$ & $\Sigma 42$ & $\Sigma 29$ & $0.67 \pm 0.17$ & $0.46 \pm 0.07$ & $0.99 \pm 0.00$ & $0.99 \pm 0.00$ & $\Sigma 94$ & $\Sigma 92$ \\
\hline$\sum \hat{\delta}$ and $q$ & $\Sigma 12472$ & $\sum 12558$ & $\sum 80^{\mathrm{A}}$ & $\sum 74^{\mathrm{A}}$ & $0.64 \pm 0.12^{A^{\prime}}$ & $0.59 \pm 0.15^{\mathrm{A}^{\prime}}$ & $0.98 \pm 0.00 \mathrm{~A}^{\mathrm{A}}$ & $0.99 \pm 0.00 \mathrm{~A}^{\prime \prime}$ & $\Sigma 202$ & $\sum 168$ \\
\hline \multicolumn{11}{|c|}{ THALS - Tincture of H. annuus L. seeds $\left(1,500 \mathrm{mg} \cdot \mathrm{Kg}^{-1}\right)$} \\
\hline$q_{1}$ & 2057 & 2171 & 13 & 17 & 0.63 & 0.78 & 0.98 & 0.98 & 42 & 39 \\
\hline$q_{2}$ & 2061 & 2063 & 14 & 18 & 0.68 & 0.87 & 0.99 & 0.98 & 31 & 36 \\
\hline$q_{3}$ & 2026 & 2090 & 10 & 11 & 0.49 & 0.53 & 0.98 & 0.99 & 44 & 23 \\
\hline$\sum q \mathrm{AA}$ & $\sum 6144$ & $\Sigma 6324$ & $\sum 37$ & $\Sigma 46$ & $0.60 \pm 0.10$ & $0.73 \pm 0.18$ & $0.98 \pm 0.00$ & $0.98 \pm 0.00$ & $\Sigma 117$ & $\sum 98$ \\
\hline$\partial_{1}$ & 2075 & 2048 & 14 & 12 & 0.67 & 0.59 & 0.98 & 0.98 & 45 & 48 \\
\hline$\hat{O}_{2}$ & 2063 & 2076 & 13 & 8 & 0.63 & 0.39 & 0.97 & 0.99 & 58 & 24 \\
\hline$\delta_{3}$ & 2068 & 2079 & 17 & 15 & 0.82 & 0.72 & 0.98 & 0.99 & 41 & 31 \\
\hline$\sum \sigma^{A A}$ & $\sum 6206$ & $\Sigma 6203$ & $\sum 44$ & $\Sigma 35$ & $0.71 \pm 0.10$ & $0.56 \pm 0.17$ & $0.98 \pm 0.00$ & $0.98 \pm 0.01$ & $\Sigma 144$ & $\Sigma 103$ \\
\hline$\Sigma \hat{o}$ and $q$ & $\Sigma 12350$ & $\sum 12527$ & $\sum 81^{\mathrm{A}}$ & $\sum 81^{A}$ & $0.66 \pm 0.11^{A^{\prime}}$ & $0.65 \pm 0.18^{\mathrm{A}^{\prime}}$ & $0.98 \pm 0.00 \mathrm{~A}^{\prime \prime}$ & $0.98 \pm 0.00 \mathrm{~A}^{\prime \prime}$ & $\Sigma 261$ & $\Sigma 201$ \\
\hline \multicolumn{11}{|c|}{ THALS - Tincture of H. annuus L. seeds $\left(2,000 \mathrm{mg} \cdot \mathrm{kg}^{-1}\right)$} \\
\hline$q_{1}$ & 2055 & 2061 & 15 & 14 & 0.73 & 0.68 & 0.97 & 0.98 & 59 & 39 \\
\hline $\mathrm{q}_{2}$ & 2052 & 2060 & 17 & 19 & 0.83 & 0.92 & 0.97 & 0.98 & 62 & 40 \\
\hline$q_{3}$ & 2079 & 2061 & 15 & 15 & 0.72 & 0.73 & 0.98 & 0.98 & 35 & 39 \\
\hline$\sum q \mathrm{AA}$ & $\sum 6186$ & $\Sigma 6182$ & $\sum 47$ & $\sum 48$ & $0.76 \pm 0.06$ & $0.78 \pm 0.13$ & $0.98 \pm 0.01$ & $0.98 \pm 0.00$ & $\sum 156$ & $\sum 118$ \\
\hline$\hat{o}_{1}$ & 2145 & 2071 & 22 & 10 & 1.03 & 0.48 & 0.97 & 0.99 & 58 & 29 \\
\hline$\hat{O}_{2}$ & 2064 & 2028 & 8 & 12 & 0.39 & 0.59 & 0.98 & 0.97 & 40 & 72 \\
\hline$\hat{o}_{3}$ & 2047 & 2071 & 18 & 15 & 0.88 & 0.72 & 0.97 & 0.99 & 56 & 29 \\
\hline$\sum \hat{\sigma} A A$ & $\Sigma 6256$ & $\Sigma 6170$ & $\sum 48$ & $\sum 37$ & $0.76 \pm 0.33$ & $0.60 \pm 0.12$ & $0.98 \pm 0.00$ & $0.98 \pm 0.01$ & $\sum 154$ & $\sum 130$ \\
\hline$\sum \hat{\sigma}$ and $q$ & $\sum 12442$ & $\Sigma 12352$ & $\sum 95^{\mathrm{A}}$ & $\sum 85^{\mathrm{A}}$ & $0.76 \pm 0.21^{A^{\prime}}$ & $0.69 \pm 0.15^{\mathrm{A}^{\prime}}$ & $0.98 \pm 0.01 \mathrm{~A}^{\prime \prime}$ & $0.98 \pm 0.01 \mathrm{~A}^{\prime \prime}$ & $\Sigma 310$ & $\sum 248$ \\
\hline \multicolumn{11}{|c|}{ THALS $\left(2 \mathrm{~g} \cdot \mathrm{kg}^{-1}\right)+$ NEU $\left(50 \mathrm{mg} \cdot \mathrm{Kg}^{-1}\right)$} \\
\hline$q_{1}$ & 2074 & 2048 & 27 & 32 & 1.30 & 1.56 & 0.99 & 0.85 & 26 & 352 \\
\hline$q_{2}$ & 2070 & 2076 & 30 & 27 & 1.45 & 1.30 & 0.99 & 0.99 & 30 & 24 \\
\hline$q_{3}$ & 2079 & 2083 & 32 & 33 & 1.54 & 1.58 & 0.99 & 0.99 & 21 & 17 \\
\hline
\end{tabular}


Table 1 The incidence of MNPCEs and PCE/NCE ratio in bone marrow of male and female Swiss albinus mice after testing for $24 \mathrm{~h}$ and $\mathbf{4 8} \mathrm{h}$ (Continued)

\begin{tabular}{|c|c|c|c|c|c|c|c|c|c|c|}
\hline$\overline{\sum q^{A A}}$ & $\sum 6223$ & $\sum 6207$ & $\sum 89$ & $\sum 92$ & $1.43 \pm 0.12$ & $1.48 \pm 0.16$ & $0.99 \pm 0.00$ & $0.94 \pm 0.08$ & $\sum 77$ & $\sum 393$ \\
\hline$\partial_{1}$ & 2077 & 2076 & 35 & 37 & 1.69 & 1.78 & 0.99 & 0.99 & 23 & 24 \\
\hline$\hat{o}_{2}$ & 2075 & 2078 & 36 & 35 & 1.73 & 1.68 & 0.99 & 0.99 & 25 & 22 \\
\hline$\hat{o}_{3}$ & 2077 & 2074 & 32 & 37 & 1.54 & 1.78 & 0.99 & 0.99 & 23 & 26 \\
\hline$\sum \sigma^{A A}$ & $\sum 6229$ & $\sum 6228$ & $\sum 103$ & $\sum 109$ & $1.65 \pm 0.10$ & $1.75 \pm 0.06$ & $0.99 \pm 0.00$ & $0.99 \pm 0.00$ & $\sum 71$ & $\sum 72$ \\
\hline$\sum \widehat{\delta}$ and $q$ & $\Sigma 12452$ & $\Sigma 12435$ & $\sum 192^{\mathrm{B}}$ & $\sum 201^{B}$ & $1.54 \pm 0.16^{B^{\prime}}$ & $1.62 \pm 0.18^{B^{\prime}}$ & $0.99 \pm 0.00 \mathrm{~A}^{\prime \prime}$ & $0.97 \pm 0.06^{\mathrm{A}^{\prime \prime}}$ & $\sum 148$ & $\sum 465$ \\
\hline \multicolumn{11}{|c|}{ THALS $\left(2 \mathrm{~g} \cdot \mathrm{kg}^{-1}\right)+$ DXR $\left(5 \mathrm{mg} \cdot \mathrm{Kg}^{-1}\right)$} \\
\hline$q_{1}$ & 2074 & 2075 & 36 & 38 & 1.74 & 1.83 & 0.99 & 0.99 & 26 & 25 \\
\hline $\mathrm{q}_{2}$ & 2075 & 2079 & 36 & 29 & 1.73 & 1.39 & 0.99 & 0.99 & 25 & 21 \\
\hline$q_{3}$ & 2074 & 2082 & 34 & 36 & 1.64 & 1.73 & 0.99 & 0.99 & 26 & 18 \\
\hline$\Sigma q^{A A}$ & $\sum 6223$ & $\sum 6236$ & $\sum 106$ & $\sum 103$ & $1.70 \pm 0.06$ & $1.65 \pm 0.23$ & $0.99 \pm 0.00$ & $0.99 \pm 0.00$ & $\sum 77$ & $\Sigma 64$ \\
\hline$\hat{o}_{1}$ & 2080 & 2089 & 34 & 28 & 1.63 & 1.34 & 0.99 & 0.99 & 20 & 11 \\
\hline$\hat{d}_{2}$ & 2081 & 2077 & 30 & 34 & 1.44 & 1.64 & 0.99 & 0.99 & 19 & 23 \\
\hline$\hat{\partial}_{3}$ & 2090 & 2082 & 33 & 34 & 1.58 & 1.63 & 1.00 & 0.99 & 10 & 18 \\
\hline$\Sigma \sigma^{A A}$ & $\sum 6251$ & $\sum 6248$ & $\sum 97$ & $\sum 96$ & $1.55 \pm 0.10$ & $1.54 \pm 0.17$ & $0.99 \pm 0.00$ & $0.99 \pm 0.00$ & $\sum 49$ & $\Sigma 52$ \\
\hline$\sum \hat{o}$ and $q$ & $\sum 12474$ & $\sum 12484$ & $\sum 203^{B}$ & $\sum 199^{B}$ & $1.63 \pm 0.11^{B^{\prime}}$ & $1.59 \pm 0.19^{B^{\prime}}$ & $0.99 \pm 0.00 \mathrm{~A}^{\prime \prime}$ & $0.99 \pm 0.00 \mathrm{~A}^{\prime \prime}$ & $\sum 126$ & $\sum 116$ \\
\hline
\end{tabular}

Means with the same letter are not significantly different $(p<0.05)$.

Shown are data from the controls ( $\mathrm{NaCl}, \mathrm{NEU}$ and DXR), an evaluation of the genotoxicity of THALS, and an evaluation of the antigenotoxicity of THALS (THALS + NEU and THALS + DXR).

specific DNA-adducts. Such results were in general agreement with evidence from experimental and epidemiological studies summarized by Bartsch and collaborators (1999) [38]: n-PUFAs are related to the generation of oxidative DNA damage, a high intake of $n-6$ PUFAs is implicated in some types of cancers, and n-9 MUFAs and n-3 PUFAs may have a role in cancer prevention. Additionally, it was suggested that the relative concentrations of short-chain C18:3n-3 linolenic acid, C18:2 n-6 linoleic acid, and polyphenols are the major factors responsible for the genotoxicity of cooking oils in the SMART assay [30]. Despite the existence of this information, contradictory or inconclusive data were found in the literature. For instance, one study reported that linoleic acid (C18:2 n-6 PUFA) suppressed cancer cell proliferation [39], while other studies indicated an enhancing effect on carcinogenesis [40,41]. Oleic acid (C18:1, n-9 MUFA), a promoter of cancer cell proliferation [39], has also been reported to be an effective anticancer and antigenotoxic agent $[42,43]$. Linolenic acid (C18:3 short-chain n-3 PUFA) had anticancer activity in some studies $[39,44]$, but promoted cancer in other studies $[41,45]$. Phenolic compounds, another important constituent of vegetable oils, are present in the unsaponifiable lipid phase. Phenolics are involved in both extra- and intracellular processes, inducing cytosolic detoxifying mechanisms, microsomal enzyme activation, and the scavenging of free radicals $[46,47]$. Evidence indicates that polyphenols can inhibit the genotoxicity of genotoxic agents $[48,49]$ and function as anticancer agents [50].

The clastogenic and cytotoxic effects from heated sunflower oil were studied in lymphocytes, hepatocytes (HepG2) and in human umbilical vein endothelial cells (HUVEC) [19]. In lymphocytes incubated with water extract of heated sunflower oil containing 0.075 or $0.15 \mu \mathrm{M}$ of thiobarbituric acid-reactive substances (this extract has a high content in polar aldehydes), the rate of chromosomal breakage was $18.4 \%$ and $23.1 \%$, compared to $8.7 \%$ and $6.6 \%$, or $8.1 \%$ and $9.2 \%$, respectively in lymphocytes incubated with the same volume of a water extract from non-heated oil or distilled water. In HepG2 or HUVEC cells, the cytotoxic properties of heated sunflower oil were dose dependent, and the cytotoxicity occurred at concentrations as low as $0.25 \mu \mathrm{M}$. In contrast, the same volume of nonheated oil or distilled water was non-toxic for these cells. The results show that a water extract obtained from heated oil is clastogenic and, in higher doses, cytotoxic. These data also suggested that a water extract, obtained from culinary oils submitted to heat stress, with a high content of aldehydes is clastogenic. It was speculated that the ingestion of large amounts of these products may also impact human health, especially in those diseases secondary to chromosomal breakage such as certain congenital malformations and certain types of cancer. This last fact can be corroborated by previous reports indicating that the administration 
Table 2 The incidence of MNPCEs and PCE/NCE ratio in bone marrow of male and female Swiss albinus mice after testing for $24 \mathrm{~h}$ and $48 \mathrm{~h}$

\begin{tabular}{|c|c|c|c|c|c|c|c|c|c|c|}
\hline \multirow[t]{2}{*}{ Treatment } & \multicolumn{2}{|c|}{ Number of PCEs analyzed } & \multicolumn{4}{|c|}{ PCEMNs } & \multicolumn{2}{|c|}{$\mathrm{PCE} /(\mathrm{PCE}+\mathrm{NCE})$} & \multicolumn{2}{|l|}{ NCE $(n)$} \\
\hline & $24 \mathrm{~h}$ & $48 \mathrm{~h}$ & $\begin{array}{l}24 \mathrm{~h} \\
(n)^{\mathrm{A}}\end{array}$ & $\begin{array}{l}48 \mathrm{~h} \\
(n)^{\mathrm{A}}\end{array}$ & $\begin{array}{l}24 \mathrm{~h} \\
(\%)^{A^{\prime}}\end{array}$ & $\begin{array}{l}48 \mathrm{~h} \\
(\%)^{A^{\prime}}\end{array}$ & $24 h^{A^{\prime \prime}}$ & $48 h^{B^{\prime \prime}}$ & $24 \mathrm{~h}$ & $48 \mathrm{~h}$ \\
\hline \multicolumn{11}{|c|}{$150 \mathrm{mM} \mathrm{NaCl}$} \\
\hline$q_{1}$ & 2095 & 2097 & 7 & 10 & 0.33 & 0.48 & 1.00 & 1.00 & 5 & 3 \\
\hline $\mathrm{q}_{2}$ & 2094 & 2095 & 9 & 10 & 0.43 & 0.48 & 1.00 & 1.00 & 6 & 5 \\
\hline$q_{3}$ & 2087 & 2089 & 11 & 8 & 0.53 & 0.38 & 0.99 & 0.99 & 13 & 11 \\
\hline$\Sigma q$ & $\sum 6276$ & $\sum 6281$ & $\Sigma 27$ & $\Sigma 28$ & $0.43 \pm 0.10$ & $0.45 \pm 0.05$ & $1.00 \pm 0.00$ & $1.00 \pm 0.00$ & $\Sigma 24$ & $\Sigma 19$ \\
\hline$\hat{o}_{1}$ & 2095 & 2088 & 9 & 13 & 0.43 & 0.62 & 1.00 & 0.99 & 5 & 12 \\
\hline$\hat{o}_{2}$ & 2055 & 2088 & 12 & 11 & 0.58 & 0.53 & 0.98 & 0.99 & 45 & 12 \\
\hline$\partial_{3}$ & 2058 & 2084 & 7 & 11 & 0.34 & 0.53 & 0.98 & 0.99 & 42 & 16 \\
\hline$\Sigma \delta$ & $\sum 6208$ & $\sum 6260$ & $\sum 28$ & $\Sigma 35$ & $0.45 \pm 0.12$ & $0.56 \pm 0.06$ & $0.99 \pm 0.01$ & $0.99 \pm 0.00$ & $\Sigma 92$ & $\sum 40$ \\
\hline$\sum \hat{o}$ and $q$ & $\sum 12484$ & $\sum 12541$ & $\sum 55^{\mathrm{A}}$ & $\sum 63^{A}$ & $0.44 \pm 0.08^{\mathrm{A}^{\prime}}$ & $0.50 \pm 0.06^{A^{\prime}}$ & $0.99 \pm 0.01 \mathrm{~A}^{\prime \prime}$ & $1.00 \pm 0.00 \mathrm{~A}^{\prime \prime}$ & $\Sigma 116$ & $\Sigma 59$ \\
\hline \multicolumn{11}{|c|}{ N-Nitroso-N-ethylurea - NEU (50 mg. $\left.\mathrm{Kg}^{-1}\right)$} \\
\hline$q_{1}$ & 2148 & 2075 & 38 & 36 & 1.77 & 1.73 & 0.49 & 0.65 & 2252 & 1125 \\
\hline$q_{2}$ & 1884 & 2032 & 32 & 34 & 1.70 & 1.67 & 0.54 & 0.81 & 1616 & 468 \\
\hline $\mathrm{q}_{3}$ & 2002 & 1948 & 15 & 31 & 0.75 & 1.59 & 0.61 & 0.93 & 1298 & 152 \\
\hline$\Sigma q$ & $\sum 6034$ & $\sum 6055$ & $\Sigma 85$ & $\Sigma 101$ & $1.41 \pm 0.57$ & $1.67 \pm 0.07$ & $0.54 \pm 0.06$ & $0.80 \pm 0.14$ & $\sum 5166$ & $\sum 1745$ \\
\hline$\partial_{1}$ & 2025 & 1999 & 64 & 31 & 3.16 & 1.55 & 0.41 & 0.36 & 2875 & 3501 \\
\hline$\hat{o}_{2}$ & 2028 & 1916 & 105 & 40 & 5.18 & 2.09 & 0.51 & 0.55 & 1972 & 1584 \\
\hline$\hat{o}_{3}$ & 2004 & 2069 & 25 & 38 & 1.25 & 1.84 & 0.67 & 0.65 & 996 & 1131 \\
\hline$\Sigma \sigma^{\pi}$ & $\sum 6057$ & $\sum 5984$ & $\sum 194$ & $\Sigma 109$ & $3.20 \pm 1.97$ & $1.83 \pm 0.27$ & $0.53 \pm 0.13$ & $0.52 \pm 0.14$ & $\Sigma 5843$ & $\Sigma 6216$ \\
\hline$\sum \hat{o}$ and $q$ & $\sum 12091$ & $\sum 12039$ & $\sum 279^{B}$ & $\sum 210^{B}$ & $2.30 \pm 1.66^{B^{\prime}}$ & $1.75 \pm 0.18^{B^{\prime}}$ & $0.54 \pm 0.06^{C^{\prime \prime}}$ & $0.66 \pm 0.16^{C^{\prime \prime}}$ & $\sum 11009$ & $\sum 7961$ \\
\hline \multicolumn{11}{|c|}{ Doxorubicin hydrochloride - DXR $\left(5 \mathrm{mg} \cdot \mathrm{Kg}^{-1}\right)$} \\
\hline$q_{1}$ & 2091 & 2017 & 49 & 36 & 2.34 & 1.78 & 0.72 & 0.96 & 809 & 83 \\
\hline $\mathrm{q}_{2}$ & 2106 & 2077 & 73 & 63 & 3.47 & 3.03 & 0.98 & 0.99 & 44 & 23 \\
\hline$q_{3}$ & 2056 & 2092 & 57 & 50 & 2.77 & 2.39 & 0.84 & 0.95 & 394 & 108 \\
\hline$\Sigma$ 우 & $\sum 6253$ & $\sum 6186$ & $\sum 179$ & $\sum 149$ & $2.86 \pm 0.57$ & $2.40 \pm 0.62$ & $0.85 \pm 0.13$ & $0.97 \pm 0.02$ & $\sum 1247$ & $\sum 214$ \\
\hline$\partial_{1}$ & 2067 & 2086 & 53 & 61 & 2.56 & 2.92 & 0.98 & 0.95 & 33 & 114 \\
\hline$\hat{o}_{2}$ & 2063 & 2042 & 56 & 70 & 2.71 & 3.43 & 0.98 & 0.97 & 37 & 58 \\
\hline$\hat{o}_{3}$ & 2082 & 2075 & 46 & 50 & 2.21 & 2.41 & 0.99 & 0.99 & 18 & 25 \\
\hline$\Sigma \delta$ & $\sum 6212$ & $\sum 6203$ & $\sum 155$ & $\sum 181$ & $2.50 \pm 0.26$ & $2.92 \pm 0.51$ & $0.99 \pm 0.00$ & $0.97 \pm 0.02$ & $\sum 88$ & $\sum 197$ \\
\hline$\sum \delta$ and $q$ & $\Sigma 12465$ & $\Sigma 12389$ & $\sum 334^{c}$ & $330^{c}$ & $2.68 \pm 0.42^{C^{\prime}}$ & $2.66 \pm 0.43^{C^{\prime}}$ & $0.92 \pm 0.07 \mathrm{~A}^{\prime \prime}$ & $0.97 \pm 0.01 \mathrm{~A}^{\prime \prime}$ & $\sum 1335$ & $\sum 411$ \\
\hline \multicolumn{11}{|c|}{ POHALS - Pharmaceutical oil of H. annuus L. seeds (250 mg. $\mathrm{Kg}^{-1}$ ) } \\
\hline$q_{1}$ & 2081 & 2092 & 9 & 7 & 0.43 & 0.33 & 0.99 & 1.00 & 14 & 10 \\
\hline $\mathrm{q}_{2}$ & 2086 & 2087 & 5 & 8 & 0.24 & 0.38 & 0.99 & 1.00 & 13 & 9 \\
\hline$q_{3}$ & 2090 & 2084 & 8 & 8 & 0.38 & 0.38 & 1.00 & 0.99 & 7 & 12 \\
\hline$\sum q^{A A}$ & $\Sigma 6257$ & $\Sigma 6263$ & $\Sigma 22$ & $\Sigma 23$ & $0.35 \pm 0.10$ & $0.37 \pm 0.03$ & $0.99 \pm 0.00$ & $1.00 \pm 0.00$ & $\Sigma 34$ & $\sum 31$ \\
\hline$\partial_{1}$ & 2082 & 2083 & 10 & 16 & 0.48 & 0.77 & 0.99 & 0.99 & 18 & 11 \\
\hline$\hat{O}_{2}$ & 2085 & 2099 & 7 & 9 & 0.34 & 0.43 & 0.99 & 1.00 & 15 & 9 \\
\hline$\partial_{3}$ & 2089 & 2072 & 9 & 15 & 0.43 & 0.72 & 0.99 & 0.99 & 11 & 21 \\
\hline$\Sigma \sigma^{B A}$ & $\Sigma 6256$ & $\Sigma 6254$ & $\Sigma 26$ & $\Sigma 40$ & $0.42 \pm 0.07$ & $0.64 \pm 0.18$ & $0.99 \pm 0.00$ & $0.99 \pm 0.00$ & $\sum 44$ & $\sum 41$ \\
\hline$\sum \widehat{O}$ and $\rho$ & $\Sigma 12513$ & $\Sigma 12517$ & $\sum 48^{A}$ & $\sum 63^{A}$ & $0.38 \pm 0.099^{A^{\prime}}$ & $0.50 \pm 0.19^{A^{\prime}}$ & $0.99 \pm 0.00 \mathrm{~A}^{\prime \prime}$ & $0.99 \pm 0.00 \mathrm{~A}^{\mathrm{A}}$ & $\sum 78$ & $\Sigma 72$ \\
\hline
\end{tabular}


Table 2 The incidence of MNPCEs and PCE/NCE ratio in bone marrow of male and female Swiss albinus mice after testing for $24 \mathrm{~h}$ and $\mathbf{4 8} \mathrm{h}$ (Continued)

\begin{tabular}{|c|c|c|c|c|c|c|c|c|c|c|}
\hline \multicolumn{11}{|c|}{ POHALS - Pharmaceutical oil of H. annuus L. seeds $\left(500 \mathrm{mg}^{\mathrm{Kg}}{ }^{-1}\right)$} \\
\hline$q_{1}$ & 2021 & 2075 & 6 & 6 & 0.30 & 0.29 & 1.00 & 0.99 & 9 & 13 \\
\hline$q_{2}$ & 2047 & 2087 & 9 & 11 & 0.44 & 0.53 & 0.99 & 1.00 & 11 & 8 \\
\hline$q_{3}$ & 2034 & 2089 & 8 & 7 & 0.39 & 0.34 & 0.99 & 0.99 & 14 & 11 \\
\hline$\sum q^{A A}$ & $\Sigma 6102$ & $\sum 6251$ & $\Sigma 23$ & $\Sigma 24$ & $0.38 \pm 0.07$ & $0.38 \pm 0.13$ & $0.99 \pm 0.00$ & $0.99 \pm 0.00$ & $\Sigma 34$ & $\Sigma 32$ \\
\hline$\partial_{1}$ & 2055 & 2057 & 10 & 11 & 0.49 & 0.53 & 0.99 & 0.99 & 28 & 18 \\
\hline$\hat{O}_{2}$ & 2067 & 2071 & 18 & 15 & 0.87 & 0.72 & 0.99 & 0.99 & 11 & 16 \\
\hline$\partial_{3}$ & 2076 & 2082 & 16 & 19 & 0.77 & 0.91 & 1.00 & 0.99 & 7 & 12 \\
\hline$\Sigma \delta^{\pi B A}$ & $\Sigma 6198$ & $\Sigma 6210$ & $\sum 44$ & $\Sigma 45$ & $0.71 \pm 0.20$ & $0.72 \pm 0.19$ & $0.99 \pm 0.01$ & $0.99 \pm 0.00$ & $\sum 46$ & $\sum 46$ \\
\hline$\sum \hat{\delta}$ and $\phi$ & $\Sigma 12300$ & $\Sigma 12461$ & $\sum 67^{A}$ & $\sum 69^{A}$ & $0.54 \pm 0.24^{\mathrm{A}^{\prime}}$ & $0.55 \pm 0.24^{\mathrm{A}^{\prime}}$ & $0.99 \pm 0.00 \mathrm{~A}^{\prime \prime}$ & $0.99 \pm 0.00 \mathrm{~A}^{\prime \prime}$ & $\Sigma 80$ & $\Sigma 78$ \\
\hline \multicolumn{11}{|c|}{ POHALS - Pharmaceutical oil of H. annuus L. seeds (1,000 mg. $\left.\mathrm{Kg}^{-1}\right)$} \\
\hline$q_{1}$ & 2088 & 2091 & 15 & 11 & 0.72 & 0.53 & 0.99 & 1.00 & 12 & 7 \\
\hline $\mathrm{q}_{2}$ & 2084 & 2086 & 9 & 16 & 0.43 & 0.77 & 0.99 & 0.99 & 14 & 14 \\
\hline$q_{3}$ & 2090 & 2080 & 11 & 9 & 0.53 & 0.43 & 1.00 & 0.99 & 10 & 11 \\
\hline$\sum q^{A A}$ & $\sum 6262$ & $\Sigma 6257$ & $\Sigma 35$ & $\Sigma 36$ & $0.56 \pm 0.15$ & $0.58 \pm 0.17$ & $0.99 \pm 0.00$ & $0.99 \pm 0.00$ & $\Sigma 36$ & $\Sigma 32$ \\
\hline$\partial_{1}$ & 2071 & 2077 & 14 & 23 & 0.68 & 1.11 & 0.99 & 0.99 & 18 & 18 \\
\hline$\hat{\partial}_{2}$ & 2087 & 2093 & 21 & 15 & 1.01 & 0.72 & 0.99 & 0.99 & 13 & 11 \\
\hline$\hat{\sigma}_{3}$ & 2084 & 2079 & 17 & 15 & 0.82 & 0.72 & 0.99 & 0.99 & 16 & 17 \\
\hline$\Sigma \delta^{B A}$ & $\sum 6242$ & $\Sigma 6249$ & $\Sigma 52$ & $\Sigma 53$ & $0.83 \pm 0.17$ & $0.85 \pm 0.22$ & $0.99 \pm 0.00$ & $0.99 \pm 0.00$ & $\sum 47$ & $\sum 46$ \\
\hline$\sum \widehat{\delta}$ and $q$ & $\Sigma 12504$ & $\Sigma 12506$ & $\sum 87^{A}$ & $\sum 89^{\mathrm{A}}$ & $0.70 \pm 0.20^{A^{\prime}}$ & $0.71 \pm 0.23^{A^{\prime}}$ & $0.99 \pm 0.00 \mathrm{~A}^{\prime \prime}$ & $0.99 \pm 0.00 \mathrm{~A}^{\prime \prime}$ & $\Sigma 83$ & $\sum 78$ \\
\hline \multicolumn{11}{|c|}{ POHALS - Pharmaceutical oil of H. annuus L. seeds $\left(1,500 \mathrm{mg} \cdot \mathrm{Kg}^{-1}\right)$} \\
\hline$q_{1}$ & 2091 & 2088 & 15 & 11 & 0.72 & 0.53 & 1.00 & 0.99 & 10 & 12 \\
\hline $\mathrm{q}_{2}$ & 2091 & 2102 & 10 & 15 & 0.48 & 0.71 & 1.00 & 1.00 & 9 & 8 \\
\hline$q_{3}$ & 2084 & 2076 & 12 & 12 & 0.58 & 0.58 & 0.99 & 0.99 & 19 & 14 \\
\hline$\sum q^{A A}$ & $\Sigma 6266$ & $\Sigma 6266$ & $\Sigma 37$ & $\Sigma 38$ & $0.59 \pm 0.12$ & $0.61 \pm 0.10$ & $0.99 \pm 0.00$ & $0.99 \pm 0.00$ & $\Sigma 38$ & $\Sigma 34$ \\
\hline$\partial_{1}$ & 2079 & 2084 & 21 & 20 & 1.01 & 0.96 & 0.99 & 0.99 & 21 & 16 \\
\hline$\hat{O}_{2}$ & 2083 & 2067 & 18 & 17 & 0.86 & 0.82 & 0.99 & 0.99 & 19 & 21 \\
\hline$\partial_{3}$ & 2091 & 2085 & 17 & 21 & 0.81 & 1.01 & 0.99 & 0.99 & 18 & 15 \\
\hline$\sum \hat{\sigma}^{B A}$ & $\Sigma 6253$ & $\Sigma 6236$ & $\Sigma 56$ & $\Sigma 58$ & $0.90 \pm 0.10$ & $0.93 \pm 0.10$ & $0.99 \pm 0.00$ & $0.99 \pm 0.00$ & $\Sigma 58$ & $\Sigma 52$ \\
\hline$\sum \delta^{\lambda}$ and $\phi$ & $\sum 12519$ & $\sum 12502$ & $\sum 93^{A}$ & $\sum 96^{\mathrm{A}}$ & $0.74 \pm 0.19^{A^{\prime}}$ & $0.77 \pm 0.20^{A^{\prime}}$ & $0.99 \pm 0.00 \mathrm{~A}^{\prime \prime}$ & $0.99 \pm 0.00 \mathrm{~A}^{\prime \prime}$ & $\sum 96$ & $\sum 86$ \\
\hline \multicolumn{11}{|c|}{ POHALS - Pharmaceutical oil of H. annuus L. seeds $\left(2,000 \mathrm{mg} \cdot \mathrm{kg}^{-1}\right)$} \\
\hline$q_{1}$ & 2084 & 2091 & 18 & 17 & 0.86 & 0.81 & 0.99 & 1.00 & 17 & 9 \\
\hline $\mathrm{P}_{2}$ & 2089 & 2087 & 16 & 18 & 0.77 & 0.86 & 0.99 & 0.99 & 15 & 13 \\
\hline$q_{3}$ & 2091 & 2085 & 9 & 13 & 0.43 & 0.62 & 0.99 & 0.99 & 11 & 15 \\
\hline$\sum q^{\mathrm{AA}}$ & $\sum 6264$ & $\Sigma 6263$ & $\Sigma 43$ & $\Sigma 48$ & $0.69 \pm 0.23$ & $0.77 \pm 0.13$ & $0.99 \pm 0.00$ & $0.99 \pm 0.00$ & $\Sigma 43$ & $\Sigma 37$ \\
\hline$\hat{o}_{1}$ & 2071 & 2074 & 24 & 25 & 1.16 & 1.21 & 0.98 & 0.98 & 36 & 32 \\
\hline$\hat{O}_{2}$ & 2085 & 2086 & 18 & 19 & 0.86 & 0.91 & 0.98 & 0.99 & 33 & 28 \\
\hline$\partial_{3}$ & 2048 & 2078 & 15 & 18 & 0.73 & 0.87 & 0.99 & 0.99 & 27 & 30 \\
\hline$\Sigma \delta^{B A}$ & $\Sigma 6204$ & $\Sigma 6238$ & $\Sigma 57$ & $\Sigma 62$ & $0.92 \pm 0.22$ & $0.99 \pm 0.18$ & $0.98 \pm 0.00$ & $0.99 \pm 0.00$ & $\Sigma 96$ & $\Sigma 90$ \\
\hline$\Sigma \hat{o}$ and $q$ & $\sum 12468$ & $\sum 12501$ & $\sum 100^{\mathrm{A}}$ & $\Sigma 110^{\mathrm{A}}$ & $0.80 \pm 0.24^{A^{\prime}}$ & $0.88 \pm 0.19^{A^{\prime}}$ & $0.99 \pm 0.00 \mathrm{~A}^{\prime \prime}$ & $0.99 \pm 0.00 \mathrm{~A}^{\prime \prime}$ & $\sum 139$ & $\Sigma 127$ \\
\hline \multicolumn{11}{|c|}{ POHALS $\left(2 \mathrm{~g} \cdot \mathrm{kg}^{-1}\right)+$ NEU $\left(50 \mathrm{mg} \cdot \mathrm{Kg}^{-1}\right)$} \\
\hline$q_{1}$ & 2040 & 2054 & 67 & 51 & 3.28 & 2.48 & 0.73 & 0.68 & 760 & 946 \\
\hline $\mathrm{q}_{2}$ & 2042 & 2068 & 61 & 61 & 2.99 & 2.95 & 0.73 & 0.65 & 758 & 1132 \\
\hline$q_{3}$ & 2039 & 2007 & 54 & 64 & 2.65 & 3.19 & 0.70 & 0.69 & 861 & 893 \\
\hline
\end{tabular}


Table 2 The incidence of MNPCEs and PCE/NCE ratio in bone marrow of male and female Swiss albinus mice after testing for $24 \mathrm{~h}$ and $48 \mathrm{~h}$ (Continued)

\begin{tabular}{|c|c|c|c|c|c|c|c|c|c|c|}
\hline$\overline{\sum q^{A A}}$ & $\sum 6121$ & $\sum 6129$ & $\sum 182$ & $\sum 176$ & $2.97 \pm 0.32$ & $2.87 \pm 0.36$ & $0.72 \pm 0.01$ & $0.67 \pm 0.02$ & $\sum 2379$ & $\overline{\sum 2971}$ \\
\hline$\partial_{1}$ & 2038 & 2014 & 46 & 52 & 2.26 & 2.58 & 0.64 & 0.69 & 1162 & 886 \\
\hline$\hat{o}_{2}$ & 2011 & 2072 & 54 & 59 & 2.69 & 2.85 & 0.67 & 0.80 & 989 & 528 \\
\hline$\hat{\sigma}_{3}$ & 2008 & 2053 & 49 & 49 & 2.44 & 2.39 & 0.69 & 0.76 & 892 & 647 \\
\hline$\sum \sigma^{B A}$ & $\sum 6057$ & $\Sigma 6139$ & $\Sigma 149$ & $\Sigma 160$ & $2.46 \pm 0.21$ & $2.61 \pm 0.23$ & $0.67 \pm 0.03$ & $0.75 \pm 0.05$ & $\Sigma 3043$ & $\Sigma 2061$ \\
\hline$\Sigma \hat{\delta}$ and $q$ & $\Sigma 12178$ & $\Sigma 12268$ & $\sum 331^{c}$ & $\sum 336^{c}$ & $2.72 \pm 0.37^{C^{\prime}}$ & $2.74 \pm 0.31^{C^{\prime}}$ & $0.69 \pm 0.044^{B \prime}$ & $0.71 \pm 0.06^{B^{\prime \prime}}$ & $\Sigma 5422$ & $\Sigma 5032$ \\
\hline \multicolumn{11}{|c|}{ POHALS (2 g. $\left.\mathrm{kg}^{-1}\right)+$ DXR (5 mg. $\left.\mathrm{Kg}^{-1}\right)$} \\
\hline$q_{1}$ & 2034 & 2166 & 76 & 52 & 3.74 & 2.40 & 0.64 & 0.83 & 1166 & 434 \\
\hline $\mathrm{O}_{2}$ & 2069 & 2015 & 51 & 49 & 2.46 & 2.43 & 0.69 & 0.69 & 931 & 885 \\
\hline$q_{3}$ & 2017 & 2066 & 52 & 59 & 2.58 & 2.86 & 0.70 & 0.69 & 883 & 934 \\
\hline$\sum q^{A A}$ & $\Sigma 6120$ & $\sum 6247$ & $\Sigma 179$ & $\sum 160$ & $2.93 \pm 0.70$ & $2.56 \pm 0.25$ & $0.67 \pm 0.03$ & $0.74 \pm 0.08$ & $\Sigma 2980$ & $\Sigma 2253$ \\
\hline$\hat{o}_{1}$ & 2057 & 2017 & 53 & 55 & 2.58 & 2.73 & 0.66 & 0.78 & 1043 & 583 \\
\hline$\hat{O}_{2}$ & 2056 & 2037 & 73 & 51 & 3.55 & 2.50 & 0.59 & 0.75 & 1444 & 663 \\
\hline$\widehat{\partial}_{3}$ & 2081 & 2021 & 47 & 49 & 2.26 & 2.42 & 0.74 & 0.78 & 719 & 579 \\
\hline$\sum \delta^{B A}$ & $\sum 6194$ & $\sum 6075$ & $\sum 173$ & $\Sigma 155$ & $2.80 \pm 0.67$ & $2.55 \pm 0.16$ & $0.66 \pm 0.08$ & $0.77 \pm 0.01$ & $\sum 3206$ & $\Sigma 1825$ \\
\hline$\Sigma \widehat{o}$ and $q$ & $\Sigma 12314$ & $\Sigma 12322$ & $\Sigma 352^{c}$ & $\Sigma 315^{c}$ & $2.86 \pm 0.62^{C^{\prime}}$ & $2.56 \pm 0.19^{C^{\prime}}$ & $0.67 \pm 0.05^{B^{\prime \prime}}$ & $0.75 \pm 0.05^{B^{\prime \prime}}$ & $\sum 6186$ & $\sum 4078$ \\
\hline
\end{tabular}

Means with the same letter are not significantly different $(p<0.05)$.

Shown are data from the controls ( $\mathrm{NaCl}, \mathrm{NEU}$ and DXR), an evaluation of the genotoxicity of POHALS, and an evaluation of the antigenotoxicity of POHALS (POHALS + NEU and POHALS + DXR).

of thermally stressed sunflower oil to rats is teratogenic [51].

Doxorubicin (DXR) is an important anthracyclines anticancer agent. It is a valuable component of various chemotherapeutic regimens for breast carcinoma and small-cell lung carcinoma. In metastatic thyroid carcinoma, DXR is most likely the best available agent [20]. However, DXR has been reported to induce micronuclei, chromatid and chromosome aberrations, and DNA single- and double-strand breaks in vitro and in vivo [52-56]. The genotoxicity of anticancer drugs is of special interest because of the risk of inducing secondary malignancies. Therefore, it is essential to screen for newer pharmacological agents that can protect the normal cells against DXR-induced cumulative (geno) toxicity. Many plants that have been widely used in traditional medicine are less toxic than pharmaceutical agents and have recently attracted the attention of researchers around the world. Plants contain many compounds, and it is likely that these can provide better protective effects than a single molecule [57]. The presence of many molecules in plants may be advantageous, as some of them may counteract the toxicity of others, and as a result, the net effect may be beneficial for therapeutic purposes. For example, the effect of various concentrations (200, 250, 300, 350, and $400 \mathrm{mg} / \mathrm{kg}$ body weight) of Aegle marmelos on the doxorubicin (DXR)-induced genotoxic effects in mice bone marrow was studied [20]. Treatment of mice with different concentrations of DXR $(5,10$, or
$15 \mathrm{mg} \cdot \mathrm{kg}^{-1}$ body weight) resulted in a dose-dependent elevation in the frequency of micronucleated polychromatic and normochromatic erythrocytes in mouse bone marrow, and it was accompanied by a DXR dosedependent decline in the PCE/NCE ratio. The treatment of mice with Aegle marmelos, orally once daily for 5 consecutive days before DXR treatment, significantly reduced the frequency of DXR-induced micronuclei and significant increased the PCE/NCE ratio at all scoring times. This observed chemoprotective effect may be due to the sum total of interaction between different ingredients of this complex mixture. The degree of protection may depend on the interaction of components individually or collectively with the genotoxic agent. The plausible mechanisms of action of Aegle marmelos in protecting against DXR-induced genomic insult were scavenging of $\mathrm{O}_{2}^{--}$and ${ }^{\circ} \mathrm{OH}$ and other free radicals, increase in antioxidant status, restoration of topoisomerase II activity, and inhibition of the formation of DXR-iron complex [20]. Another study was undertaken to evaluate the genotoxic potential of Copaifera langsdorffii Desf. leaf hydroalcoholic extract and its influence on the genotoxicity induced by chemotherapeutic agent DXR using the Swiss mouse peripheral blood micronucleus test. The results of this study demonstrated that C. langsdorffii Desf. was not itself genotoxic and that in animals treated with $C$. langsdorffii Desf. and DXR, the number of micronuclei was significantly decreased compared to animals receiving DXR alone. The putative 
Table 3 The incidence of MNPCEs and PCE/NCE ratio in bone marrow of male and female Swiss albinus mice after testing for $24 \mathrm{~h}$ and $48 \mathrm{~h}$

\begin{tabular}{|c|c|c|c|c|c|c|c|c|c|c|}
\hline \multirow[t]{2}{*}{ Treatment } & \multicolumn{2}{|c|}{ Number of PCEs analyzed } & \multicolumn{4}{|c|}{ PCEMNs } & \multicolumn{2}{|c|}{$\mathrm{PCE} /(\mathrm{PCE}+\mathrm{NCE})$} & \multicolumn{2}{|l|}{$\operatorname{NCE}(n)$} \\
\hline & $24 \mathrm{~h}$ & $48 \mathrm{~h}$ & $\begin{array}{l}24 \mathrm{~h} \\
(n)^{\mathrm{A}}\end{array}$ & $\begin{array}{l}48 \mathrm{~h} \\
(n)^{\mathrm{A}}\end{array}$ & $\begin{array}{l}24 \mathrm{~h} \\
(\%)^{\mathrm{A}^{\prime}}\end{array}$ & $\begin{array}{l}48 \mathrm{~h} \\
(\%)^{A^{\prime}}\end{array}$ & $24 \mathrm{~h} \mathrm{~A}^{\mathrm{A}}$ & $48 h^{A^{\prime \prime}}$ & $24 \mathrm{~h}$ & $48 \mathrm{~h}$ \\
\hline \multicolumn{11}{|c|}{$150 \mathrm{mM} \mathrm{NaCl}$} \\
\hline$q_{1}$ & 2095 & 2097 & 7 & 10 & 0.33 & 0.48 & 1.00 & 1.00 & 5 & 3 \\
\hline $\mathrm{q}_{2}$ & 2094 & 2095 & 9 & 10 & 0.43 & 0.48 & 1.00 & 1.00 & 6 & 5 \\
\hline$q_{3}$ & 2087 & 2089 & 11 & 8 & 0.53 & 0.38 & 0.99 & 0.99 & 13 & 11 \\
\hline$\Sigma q$ & $\Sigma 6276$ & $\sum 6281$ & $\sum 27$ & $\Sigma 28$ & $0.43 \pm 0.10$ & $0.45 \pm 0.05$ & $1.00 \pm 0.00$ & $1.00 \pm 0.00$ & $\sum 24$ & $\Sigma 19$ \\
\hline$\hat{o}_{1}$ & 2095 & 2088 & 9 & 13 & 0.43 & 0.62 & 1.00 & 0.99 & 5 & 12 \\
\hline$\hat{o}_{2}$ & 2055 & 2088 & 12 & 11 & 0.58 & 0.53 & 0.98 & 0.99 & 45 & 12 \\
\hline$\partial_{3}$ & 2058 & 2084 & 7 & 11 & 0.34 & 0.53 & 0.98 & 0.99 & 42 & 16 \\
\hline$\Sigma \delta$ & $\sum 6208$ & $\sum 6260$ & $\sum 28$ & $\Sigma 35$ & $0.45 \pm 0.12$ & $0.56 \pm 0.06$ & $0.99 \pm 0.01$ & $0.99 \pm 0.00$ & $\Sigma 92$ & $\sum 40$ \\
\hline$\sum \hat{o}$ and $q$ & $\sum 12484$ & $\sum 12541$ & $\sum 55^{\mathrm{A}}$ & $\sum 63^{A}$ & $0.44 \pm 0.08^{A^{\prime}}$ & $0.50 \pm 0.06^{\mathrm{A}^{\prime}}$ & $0.99 \pm 0.01 \mathrm{~A}^{\prime \prime}$ & $1.00 \pm 0.00 \mathrm{~A}^{\prime \prime}$ & $\sum 116$ & $\Sigma 59$ \\
\hline \multicolumn{11}{|c|}{ N-Nitroso-N-ethylurea - NEU (50 mg. $\left.\mathrm{Kg}^{-1}\right)$} \\
\hline$q_{1}$ & 2148 & 2075 & 38 & 36 & 1.77 & 1.73 & 0.49 & 0.65 & 2252 & 1125 \\
\hline$q_{2}$ & 1884 & 2032 & 32 & 34 & 1.70 & 1.67 & 0.54 & 0.81 & 1616 & 468 \\
\hline $\mathrm{q}_{3}$ & 2002 & 1948 & 15 & 31 & 0.75 & 1.59 & 0.61 & 0.93 & 1298 & 152 \\
\hline$\Sigma q$ & $\sum 6034$ & $\Sigma 6055$ & $\sum 85$ & $\sum 101$ & $1.41 \pm 0.57$ & $1.67 \pm 0.07$ & $0.54 \pm 0.06$ & $0.80 \pm 0.14$ & $\sum 5166$ & $\sum 1745$ \\
\hline$\partial_{1}$ & 2025 & 1999 & 64 & 31 & 3.16 & 1.55 & 0.41 & 0.36 & 2875 & 3501 \\
\hline$\hat{o}_{2}$ & 2028 & 1916 & 105 & 40 & 5.18 & 2.09 & 0.51 & 0.55 & 1972 & 1584 \\
\hline$\hat{\sigma}_{3}$ & 2004 & 2069 & 25 & 38 & 1.25 & 1.84 & 0.67 & 0.65 & 996 & 1131 \\
\hline$\Sigma \sigma^{\pi}$ & $\sum 6057$ & $\sum 5984$ & $\sum 194$ & $\Sigma 109$ & $3.20 \pm 1.97$ & $1.83 \pm 0.27$ & $0.53 \pm 0.13$ & $0.52 \pm 0.14$ & $\Sigma 5843$ & $\Sigma 6216$ \\
\hline$\sum \hat{o}$ and $q$ & $\Sigma 12091$ & $\sum 12039$ & $\sum 279^{B}$ & $\sum 210^{B}$ & $2.30 \pm 1.66^{B^{\prime}}$ & $1.75 \pm 0.18^{B^{\prime}}$ & $0.54 \pm 0.06^{D^{\prime \prime}}$ & $0.66 \pm 0.16^{D^{\prime \prime}}$ & $\sum 11009$ & $\Sigma 7961$ \\
\hline \multicolumn{11}{|c|}{ Doxorubicin hydrochloride - DXR (5 mg. $\left.\mathrm{Kg}^{-1}\right)$} \\
\hline$q_{1}$ & 2091 & 2017 & 49 & 36 & 2.34 & 1.78 & 0.72 & 0.96 & 809 & 83 \\
\hline $\mathrm{q}_{2}$ & 2106 & 2077 & 73 & 63 & 3.47 & 3.03 & 0.98 & 0.99 & 44 & 23 \\
\hline$q_{3}$ & 2056 & 2092 & 57 & 50 & 2.77 & 2.39 & 0.84 & 0.95 & 394 & 108 \\
\hline$\Sigma$ 우 & $\sum 6253$ & $\sum 6186$ & $\sum 179$ & $\sum 149$ & $2.86 \pm 0.57$ & $2.40 \pm 0.62$ & $0.85 \pm 0.13$ & $0.97 \pm 0.02$ & $\sum 1247$ & $\sum 214$ \\
\hline$\partial_{1}$ & 2067 & 2086 & 53 & 61 & 2.56 & 2.92 & 0.98 & 0.95 & 33 & 114 \\
\hline$\hat{o}_{2}$ & 2063 & 2042 & 56 & 70 & 2.71 & 3.43 & 0.98 & 0.97 & 37 & 58 \\
\hline$\hat{O}_{3}$ & 2082 & 2075 & 46 & 50 & 2.21 & 2.41 & 0.99 & 0.99 & 18 & 25 \\
\hline$\Sigma \delta$ & $\sum 6212$ & $\sum 6203$ & $\sum 155$ & $\sum 181$ & $2.50 \pm 0.26$ & $2.92 \pm 0.51$ & $0.99 \pm 0.00$ & $0.97 \pm 0.02$ & $\sum 88$ & $\sum 197$ \\
\hline$\sum \delta$ and $q$ & $\sum 12465$ & $\Sigma 12389$ & $\sum 334^{c}$ & $330^{c}$ & $2.68 \pm 0.42^{C^{\prime}}$ & $2.66 \pm 0.43^{C^{\prime}}$ & $0.92 \pm 0.07 \mathrm{AB}^{\prime \prime}$ & $0.97 \pm 0.01{ }^{A B "}$ & $\sum 1335$ & $\sum 411$ \\
\hline \multicolumn{11}{|c|}{ FOHALS - Food oil of H. annuus L. seeds (250 mg. $\mathrm{kg}^{-1}$ ) } \\
\hline$q_{1}$ & 2137 & 2019 & 11 & 9 & 0.51 & 0.45 & 0.99 & 0.99 & 24 & 14 \\
\hline $\mathrm{q}_{2}$ & 2142 & 2073 & 7 & 14 & 0.33 & 0.68 & 1.00 & 1.00 & 8 & 5 \\
\hline $\mathrm{q}_{3}$ & 2146 & 2016 & 9 & 7 & 0.42 & 0.35 & 0.99 & 1.00 & 12 & 6 \\
\hline$\sum q \mathrm{AA}$ & $\sum 6425$ & $\sum 6108$ & $\sum 27$ & $\Sigma 30$ & $0.42 \pm 0.09$ & $0.49 \pm 0.17$ & $0.99 \pm 0.00$ & $1.00 \pm 0.00$ & $\sum 44$ & $\sum 25$ \\
\hline$\delta_{1}$ & 2061 & 2079 & 6 & 10 & 0.29 & 0.48 & 0.95 & 0.94 & 98 & 140 \\
\hline$\hat{O}_{2}$ & 2093 & 2093 & 8 & 7 & 0.38 & 0.33 & 0.99 & 0.99 & 31 & 20 \\
\hline$\hat{O}_{3}$ & 2050 & 2041 & 8 & 6 & 0.39 & 0.29 & 0.99 & 0.97 & 30 & 59 \\
\hline$\Sigma \overbrace{}^{B B}$ & $\Sigma 6204$ & $\Sigma 6213$ & $\Sigma 22$ & $\Sigma 23$ & $0.35 \pm 0.03$ & $0.37 \pm 0.10$ & $0.98 \pm 0.02$ & $0.97 \pm 0.03$ & $\sum 159$ & $\Sigma 219$ \\
\hline$\sum \widehat{O}$ and $\rho$ & $\Sigma 12629$ & $\Sigma 12321$ & $\sum 49^{A}$ & $\Sigma 53^{\mathrm{A}}$ & $0.39 \pm 0.08^{A^{\prime}}$ & $0.43 \pm 0.14^{A^{\prime}}$ & $0.98 \pm 0.02^{\mathrm{A}^{\prime \prime}}$ & $0.98 \pm 0.02^{\mathrm{A}^{\prime \prime}}$ & $\Sigma 203$ & $\Sigma 244$ \\
\hline
\end{tabular}


Table 3 The incidence of MNPCEs and PCE/NCE ratio in bone marrow of male and female Swiss albinus mice after testing for $\mathbf{2 4} \mathrm{h}$ and $\mathbf{4 8} \mathrm{h}$ (Continued)

\begin{tabular}{|c|c|c|c|c|c|c|c|c|c|c|}
\hline \multicolumn{11}{|c|}{ FOHALS - Food oil of H. annuus L. seeds (500 $\mathrm{mg} \mathrm{Kg}^{-1}$ ) } \\
\hline$q_{1}$ & 2014 & 2046 & 10 & 11 & 0.50 & 0.54 & 0.98 & 0.99 & 39 & 24 \\
\hline$q_{2}$ & 2007 & 2094 & 8 & 8 & 0.40 & 0.38 & 0.95 & 0.98 & 106 & 34 \\
\hline$q_{3}$ & 2010 & 2035 & 9 & 16 & 0.45 & 0.79 & 0.96 & 0.99 & 81 & 11 \\
\hline$\sum q^{\mathrm{AA}}$ & $\Sigma 6031$ & $\sum 6175$ & $\sum 27$ & $\sum 35$ & $0.45 \pm 0.05$ & $0.57 \pm 0.20$ & $0.96 \pm 0.02$ & $0.99 \pm 0.01$ & $\Sigma 226$ & $\Sigma 69$ \\
\hline$\partial_{1}$ & 2037 & 2082 & 10 & 10 & 0.49 & 0.48 & 0.95 & 0.94 & 116 & 135 \\
\hline$\hat{o}_{2}$ & 2063 & 2067 & 8 & 16 & 0.39 & 0.77 & 0.96 & 0.96 & 78 & 89 \\
\hline$\hat{O}_{3}$ & 2053 & 2078 & 5 & 9 & 0.24 & 0.43 & 0.95 & 0.97 & 102 & 63 \\
\hline$\Sigma \delta^{\lambda B}$ & $\Sigma 6153$ & $\Sigma 6227$ & $\Sigma 23$ & $\Sigma 35$ & $0.37 \pm 0.12$ & $0.56 \pm 0.18$ & $0.95 \pm 0.01$ & $0.96 \pm 0.02$ & $\Sigma 296$ & $\Sigma 287$ \\
\hline$\sum \delta$ and $\phi$ & $\sum 12184$ & $\Sigma 12402$ & $\sum 50^{A}$ & $\sum 70^{A}$ & $0.41 \pm 0.09^{A^{\prime}}$ & $0.57 \pm 0.17^{A^{\prime}}$ & $0.96 \pm 0.01{ }^{A B^{\prime \prime}}$ & $0.97 \pm 0.02^{A B^{\prime \prime}}$ & $\Sigma 522$ & $\sum 356$ \\
\hline \multicolumn{11}{|c|}{ FOHALS - Food oil of H. annuus L. seeds $\left(1,000 \mathrm{mg} \cdot \mathrm{Kg}^{-1}\right)$} \\
\hline$q_{1}$ & 2114 & 2083 & 11 & 16 & 0.52 & 0.77 & 0.98 & 0.98 & 36 & 48 \\
\hline $\mathrm{O}_{2}$ & 2148 & 2058 & 11 & 13 & 0.51 & 0.63 & 0.96 & 0.97 & 84 & 72 \\
\hline$q_{3}$ & 2097 & 2090 & 8 & 9 & 0.38 & 0.43 & 0.93 & 0.95 & 149 & 106 \\
\hline$\sum q^{\mathrm{AA}}$ & $\Sigma 6359$ & $\Sigma 6231$ & $\Sigma 30$ & $\Sigma 38$ & $0.47 \pm 0.08$ & $0.61 \pm 0.17$ & $0.96 \pm 0.02$ & $0.97 \pm 0.01$ & $\sum 269$ & $\Sigma 226$ \\
\hline$\delta_{1}$ & 2073 & 2026 & 9 & 17 & 0.43 & 0.84 & 0.90 & 0.96 & 227 & 74 \\
\hline$\hat{O}_{2}$ & 2065 & 2071 & 12 & 13 & 0.58 & 0.63 & 0.99 & 0.94 & 20 & 129 \\
\hline$\hat{o}_{3}$ & 2003 & 2084 & 5 & 8 & 0.25 & 0.38 & 0.95 & 0.91 & 97 & 207 \\
\hline$\Sigma O^{\lambda B} B$ & $\Sigma 6141$ & $\sum 6181$ & $\sum 26$ & $\sum 38$ & $0.42 \pm 0.17$ & $0.62 \pm 0.23$ & $0.95 \pm 0.02$ & $0.94 \pm 0.03$ & $\sum 344$ & $\sum 410$ \\
\hline$\Sigma \hat{o}$ and $q$ & $\Sigma 12500$ & $\Sigma 12412$ & $\sum 56^{\mathrm{A}}$ & $\sum 76^{\mathrm{A}}$ & $0.45 \pm 0.12^{A^{\prime}}$ & $0.61 \pm 0.18^{A^{\prime}}$ & $0.95 \pm 0.03^{A B^{\prime \prime}}$ & $0.95 \pm 0.02 \mathrm{AB}^{\prime \prime}$ & $\Sigma 613$ & $\Sigma 636$ \\
\hline \multicolumn{11}{|c|}{ FOHALS - Food oil of H. annuus L. seeds $\left(1,500 \mathrm{mg} \cdot \mathrm{Kg}^{-1}\right)$} \\
\hline$q_{1}$ & 2065 & 2021 & 6 & 15 & 0.29 & 0.74 & 0.98 & 0.90 & 35 & 236 \\
\hline$q_{2}$ & 2041 & 2081 & 13 & 10 & 0.64 & 0.48 & 0.93 & 0.89 & 159 & 262 \\
\hline$q_{3}$ & 2068 & 2072 & 11 & 14 & 0.53 & 0.68 & 0.89 & 0.88 & 247 & 294 \\
\hline$\sum q^{\mathrm{A} A}$ & $\sum 6174$ & $\sum 6174$ & $\Sigma 30$ & $\Sigma 39$ & $0.49 \pm 0.18$ & $0.63 \pm 0.14$ & $0.93 \pm 0.05$ & $0.89 \pm 0.01$ & $\sum 441$ & $\sum 792$ \\
\hline$\partial_{1}$ & 2106 & 2087 & 10 & 18 & 0.47 & 0.86 & 0.92 & 0.96 & 194 & 94 \\
\hline$\hat{O}_{2}$ & 2011 & 2088 & 5 & 11 & 0.25 & 0.53 & 0.91 & 0.94 & 189 & 126 \\
\hline$\hat{o}_{3}$ & 2039 & 2128 & 14 & 16 & 0.69 & 0.75 & 0.98 & 0.88 & 38 & 277 \\
\hline$\Sigma \sigma^{B B}$ & $\Sigma 6156$ & $\Sigma 6303$ & $\Sigma 29$ & $\Sigma 45$ & $0.47 \pm 0.22$ & $0.71 \pm 0.17$ & $0.94 \pm 0.04$ & $0.93 \pm 0.04$ & $\sum 421$ & $\sum 497$ \\
\hline$\sum \delta^{\lambda}$ and $\phi$ & $\sum 12330$ & $\sum 12477$ & $\sum 59^{\mathrm{A}}$ & $\sum 84^{\mathrm{A}}$ & $0.48 \pm 0.18^{\mathrm{A}^{\prime}}$ & $0.67 \pm 0.15^{A^{\prime}}$ & $0.94 \pm 0.04 \mathrm{AB}^{\prime \prime}$ & $0.91 \pm 0.033^{A B^{\prime \prime}}$ & $\sum 862$ & $\sum 1289$ \\
\hline \multicolumn{11}{|c|}{ FOHALS - Food oil of H. annuus L. seeds $\left(2,000 \mathrm{mg} \cdot \mathrm{kg}^{-1}\right)$} \\
\hline$q_{1}$ & 2084 & 2015 & 11 & 13 & 0.53 & 0.65 & 0.89 & 0.86 & 245 & 325 \\
\hline $\mathrm{P}_{2}$ & 2096 & 2025 & 13 & 11 & 0.62 & 0.54 & 0.91 & 0.83 & 216 & 407 \\
\hline$q_{3}$ & 2076 & 2002 & 9 & 17 & 0.43 & 0.85 & 0.95 & 0.94 & 114 & 126 \\
\hline$\sum q^{A A}$ & $\Sigma 6256$ & $\Sigma 6042$ & $\Sigma 33$ & $\sum 41$ & $0.53 \pm 0.09$ & $0.68 \pm 0.16$ & $0.92 \pm 0.03$ & $0.88 \pm 0.06$ & $\Sigma 575$ & $\Sigma 858$ \\
\hline$\hat{o}_{1}$ & 2057 & 2199 & 16 & 20 & 0.78 & 0.91 & 0.94 & 0.97 & 143 & 65 \\
\hline$\hat{\partial}_{2}$ & 2016 & 2158 & 17 & 12 & 0.84 & 0.56 & 0.95 & 0.86 & 110 & 347 \\
\hline$\hat{o}_{3}$ & 2106 & 2133 & 11 & 15 & 0.52 & 0.70 & 0.92 & 0.89 & 194 & 261 \\
\hline$\Sigma \delta^{B B}$ & $\Sigma 6179$ & $\Sigma 6490$ & $\Sigma 44$ & $\sum 47$ & $0.71 \pm 0.17$ & $0.72 \pm 0.18$ & $0.93 \pm 0.02$ & $0.91 \pm 0.06$ & $\sum 447$ & $\Sigma 673$ \\
\hline$\sum \sigma^{\pi}$ and $q$ & $\Sigma 12435$ & $\Sigma 12532$ & $\sum 77^{\mathrm{A}}$ & $\sum 88^{A}$ & $0.62 \pm 0.16^{\mathrm{A}^{\prime}}$ & $0.70 \pm 0.15^{A^{\prime}}$ & $0.92 \pm 0.02^{B^{\prime \prime}}$ & $0.89 \pm 0.05^{B^{\prime \prime}}$ & $\Sigma 1022$ & $\sum 1531$ \\
\hline \multicolumn{11}{|c|}{ FOHALS $\left(2 \mathrm{~g} \cdot \mathrm{kg}^{-1}\right)+$ NEU $\left(50 \mathrm{mg} \cdot \mathrm{Kg}^{-1}\right)$} \\
\hline$q_{1}$ & 2020 & 2062 & 54 & 49 & 2.67 & 2.38 & 0.70 & 0.74 & 880 & 738 \\
\hline $\mathrm{q}_{2}$ & 2080 & 2052 & 44 & 52 & 2.12 & 2.53 & 0.80 & 0.68 & 520 & 948 \\
\hline$q_{3}$ & 2008 & 2011 & 50 & 54 & 2.49 & 2.69 & 0.69 & 0.74 & 892 & 689 \\
\hline
\end{tabular}


Table 3 The incidence of MNPCEs and PCE/NCE ratio in bone marrow of male and female Swiss albinus mice after testing for $24 \mathrm{~h}$ and $48 \mathrm{~h}$ (Continued)

\begin{tabular}{|c|c|c|c|c|c|c|c|c|c|c|}
\hline$\Sigma q^{\mathrm{AA}}$ & $\Sigma 6108$ & $\Sigma 6125$ & $\Sigma 148$ & $\Sigma 155$ & $2.43 \pm 0.28$ & $2.53 \pm 0.15$ & $0.73 \pm 0.06$ & $0.72 \pm 0.03$ & $\Sigma 2292$ & $\Sigma 2375$ \\
\hline$\hat{o}_{1}$ & 2031 & 2013 & 50 & 67 & 2.46 & 3.33 & 0.73 & 0.75 & 769 & 687 \\
\hline$\hat{\sigma}_{2}$ & 2010 & 2054 & 43 & 67 & 2.14 & 3.26 & 0.65 & 0.76 & 1090 & 646 \\
\hline$\delta_{3}$ & 2042 & 2045 & 56 & 71 & 2.74 & 3.47 & 0.64 & 0.71 & 1158 & 855 \\
\hline$\sum \sigma^{B} B$ & $\Sigma 6083$ & $\sum 6112$ & $\Sigma 149$ & $\Sigma 205$ & $2.45 \pm 0.30$ & $3.35 \pm 0.11$ & $0.67 \pm 0.05$ & $0.74 \pm 0.03$ & $\sum 3017$ & $\Sigma 2188$ \\
\hline$\Sigma \delta$ and $q$ & $\sum 12191$ & $\sum 12237$ & $\Sigma 297^{C}$ & $\sum 360^{c}$ & $2.44 \pm 0.26^{C^{\prime}}$ & $2.94 \pm 0.47^{C^{\prime}}$ & $0.70 \pm 0.06^{C^{\prime \prime}}$ & $0.73 \pm 0.03^{C^{\prime \prime}}$ & $\sum 5309$ & $\sum 4563$ \\
\hline \multicolumn{11}{|c|}{ FOHALS $\left(2 \mathrm{~g} \cdot \mathrm{kg}^{-1}\right)+$ DXR $\left(5 \mathrm{mg} \cdot \mathrm{Kg}^{-1}\right)$} \\
\hline$q_{1}$ & 2007 & 2051 & 50 & 54 & 2.49 & 2.63 & 0.77 & 0.82 & 593 & 449 \\
\hline$q_{2}$ & 2010 & 2033 & 64 & 49 & 3.18 & 2.41 & 0.77 & 0.88 & 590 & 267 \\
\hline$q_{3}$ & 2094 & 2031 & 51 & 54 & 2.44 & 2.66 & 0.84 & 0.85 & 406 & 369 \\
\hline$\sum q \mathrm{AA}$ & $\sum 6111$ & $\sum 6115$ & $\sum 165$ & $\sum 157$ & $2.70 \pm 0.42$ & $2.57 \pm 0.14$ & $0.79 \pm 0.04$ & $0.85 \pm 0.03$ & $\Sigma 1589$ & $\Sigma 1085$ \\
\hline$\hat{o}_{1}$ & 2128 & 2067 & 67 & 66 & 3.15 & 3.19 & 0.85 & 0.67 & 372 & 1033 \\
\hline$\widehat{\partial}_{2}$ & 2015 & 2053 & 79 & 65 & 3.92 & 3.17 & 0.69 & 0.71 & 885 & 847 \\
\hline$\hat{\sigma}_{3}$ & 2054 & 2041 & 66 & 60 & 3.21 & 2.94 & 0.64 & 0.66 & 1146 & 1059 \\
\hline$\sum \sigma^{B B}$ & $\sum 6197$ & $\sum 6161$ & $\Sigma 212$ & $\Sigma 191$ & $3.43 \pm 0.43$ & $3.10 \pm 0.14$ & $0.73 \pm 0.11$ & $0.68 \pm 0.03$ & $\Sigma 2403$ & $\Sigma 2939$ \\
\hline$\Sigma \hat{o}$ and $q$ & $\Sigma 12308$ & $\Sigma 12276$ & $\sum 377^{C}$ & $\sum 348^{C}$ & $3.07 \pm 0.55^{C^{\prime}}$ & $2.83 \pm 0.32^{C^{\prime}}$ & $0.76 \pm 0.08^{C^{\prime \prime}}$ & $0.76 \pm 0.10^{C^{\prime \prime}}$ & $\sum 3992$ & $\sum 4024$ \\
\hline
\end{tabular}

Means with the same letter are not significantly different $(p<0.05)$.

Shown are data from the controls ( $\mathrm{NaCl}, \mathrm{NEU}$ and DXR), an evaluation of the genotoxicity of FOHALS, and an evaluation of the antigenotoxicity of FOHALS (FOHALS + NEU and FOHALS + DXR).

antioxidant activity of one or more of the active compounds of $C$. langsdorffii Desf., including two major flavonoid heterosides (quercitrin and afzelin), may explain the effect of this plant on DXR genotoxicity [18].

\section{Conclusions}

In conclusion, this research observed an absence of genotoxicity of a tincture and two oils of sunflower seeds, regardless of the dose tested and the treatment time (24-48 h), but sex-independent (sunflower tincture) or sex-dependent (sunflower oils). Antigenotoxic effects (anticlastogeny and/or antianeugeny) were observed using only a dose of the sunflower tincture in association with the chemotherapy agent DXR. Therefore, the sunflower tincture can promote a partial protection against the genotoxic effects induced by DXR. The sunflower tincture no showed systemic toxicity and it was dose- and timeindependent and sex-dependent, whereas the systemic toxicity of sunflower oil can be dependent on its source and its highest dose used.

Other studies involving the genotoxicity and antigenotoxicity of $H$. annuus L. extracts and oils (seeds, flowers and leaves) should be conducted [including genotoxicity assays with Salmonella typhimurium test (Ames test) as an indicator of potential carcinogenicity to mammals, gene mutation test in mammalian cells (mouse lymphoma assay), cytogenetic and aneuploidy tests in vitro, micronucleus test in cultured cells in vitro, fluorescent in situ hybridization (FISH) test for mutagenesis, comet test to detect of DNA damage and repair in individual cells, and functional genomic and proteomic tests for mutagenesis (cDNA microarrays and other array analyses)], to characterize the potential effects and genotoxic and antigenotoxic mechanisms and, importantly, for the establishment of limits for human consumption, the delineation of potential risks to human health, and for rational strategies for implementing chemo-preventive measures.

\section{Competing interest}

The authors have declared no competing interest.

\section{Authors' contributions}

MFGB, JEF and NMSO wrote and revised the draft, MCCR and TAS provided animals care and revised the draft, LSS and MRR aided micronucleus assays and revised the draft, MFGB and CTSD performed statistical analysis. MFGB and JEF have given final approval of the version to be published. All authors have read and approved the final manuscript.

\section{Acknowledgements}

This research was supported by Rede Mineira de Ensaios Toxicológicos e Farmacológicos de Produtos Terapêuticos (REDE MINEIRA TOXIFAR - 2012), Fundação de Amparo à Pesquisa do Estado de Minas Gerais (FAPEMIG). The authors thank the Language Services of Elsevier for help in English language editing.

\section{Author details}

${ }^{1}$ Laboratório de Farmacogenômica e Biologia Molecular, Faculdade de Ciências Médicas \& Centro de Pesquisa e Pós-graduação, Universidade José do Rosário Vellano (UNIFENAS), Campus Universitário, Rod. MG 179, Km 0, Alfenas, MG CEP: 37130-000, Brasil. ${ }^{2}$ Centro de Pesquisa e Pós-graduação em Ciência Animal, Área de Patologia e Farmacologia Animal, Universidade José do Rosário Vellano (UNIFENAS), Alfenas, Minas Gerais, Brasil. ${ }^{3}$ Centro de Cirurgia Experimental e Farmacologia, Universidade Estadual de Campinas, 
Campinas (UNICAMP), São Paulo, Brasil. ${ }^{4}$ Laboratório de Ecotoxicologia e Microbiologia Ambiental, Faculdade de Tecnologia, Universidade Estadual de Campinas (UNICAMP), Limeira, São Paulo, Brasil. . ${ }^{5}$ Departamento de Ciências Exatas, Escola de Agricultura "Luiz de Queiroz", Universidade de São Paulo (ESALQ/USP), Piracicaba, SP, Brasil.

Received: 22 August 2013 Accepted: 27 March 2014

Published: 2 April 2014

\section{References}

1. Heiser CB: Sunflowers: Helianthus (Compositae-Heliantheae). In Evolution of Crop Plants. Edited by Simmonds NW. London: Longmans Green; 1976:36-38.

2. Earle FR, Vanetten CH, Clark TF, Wolff IA: Compositional data on sunflower seed. J Am Oil Chem Soc 1968, 45:876-879.

3. Salunkhe DK, Chavan JK, Adsule RN, Kadam SS: World Oilseeds: Chemistry, Technology and Utilization. In Sunflower. New York: Van Nostrand Reinhold; 1992.

4. Gonzáles-Pérez S, Vereijken JM: Sunflower proteins: Overview of their physicochemical, structural and functional properties. J Sci Food Agric 2007, 87:2173-2191.

5. Warner KA, Mounts TL, List GVR: Effects of added tocopherols on the flavor stability of purified vegetable oils. Inform 1990, 1:326.

6. Ito T, Tamura T, Matsumoto T: Sterol Composition of 19 vegetable oils. J Am Oil Chem Soc 1973, 50:122-125.

7. Trost W: Characterization of corn oil, soybean oil and sunflower oil nonpolar material. J Am Oil Chem Soc 1989, 66:325-333.

8. Dupont HL, Sullivan P, Evans DG, Vollet JJ, Ericsson CD, Ackerman PB, Tjoa WS: Prevention of traveler's diarrhea (emporiatric enteritis). Prophylactic administration of subsalicylate bismuth. JAMA 1980, 243:237-241.

9. Cowan JC: Key factors and recent advances in the flavor stability of soybean oil. J Am Oil Chem Soc 1966, 43:300A, 302A, 318A, 320.

10. Bierenbaum ML, Fleischman Al, Dun J, Arnold J: Possible toxic waste factor in coronary heart disease. Lancet 1975, 1:1008-1010

11. Purves D, Harvey C, Tweats D, Lumley CE: Genotoxity testing: current practices and strategies used by the pharmaceutical industry. Mutagenesis 1995, 10:297-312.

12. Varanda EA: Atividade mutagênica de plantas medicinais. Rev Ciênc Farm Básica Apl 2006, 27:1-7.

13. Mateuca R, Lombaert N, Aka PV, Decordier I, Kirsch-Volders M: Chromosomal changes: induction, detection methods and applicability in human biomonitoring. Biochimie 2006, 88:1515-1531.

14. Krishna G, Hayashi M: In vivo rodent micronucleus assay: protocol, conduct and data interpretation. Mutat Res 2000, 455:155-166.

15. Organisation for Economic Cooperation and Development: OECD Guideline for the Testing of Chemicals: Bacterial reverse mutation test. Paris; 1997:Guideline 471.

16. Organisation for Economic Cooperation and Development: OECD Guideline for the Testing of Chemicals: In vitro mammalian chromosome aberration test. Paris; 1997:Guideline 473.

17. Organisation for Economic Cooperation and Development: OECD Guideline for the Testing of Chemicals: Mammalian erythrocyte micronucleus test. Paris; 1997:Guideline 474.

18. Alves JM, Munari CC, Neto MABM, Furtado RA, Senedese JM, Bastos JK, Tavares DC: In vivo protective effect of Copaifera langsdorffii hydroalcoholic extract on micronuclei induction by doxorubicin. J Appl Toxicol 2013, 33:854-860.

19. Indart A, Viana M, Clapés S, Izquierdo L, Bonet B: Clastogenic and cytotoxic effects of lipid peroxidation products generated in culinary oils submitted to thermal stress. Food Chem Toxicol 2007, 45:1963-1967.

20. Venkatesh P, Shantala B, Jagetia GC, Rao KK, Baliga MS: Modulation of Doxorubicin-Induced Genotoxicity by Aegle marmelos in Mouse Bone Marrow: A Micronucleus Study. Integr Cancer Ther 2007, 6:42-53.

21. Brasil: Farmacopéia Brasileira, Agência Nacional de Vigilância Sanitária ANVISA/Fundação Oswaldo. Brasília: Cruz - FIOCRUZ; 2010.

22. Collaborative Study Group for the Micronucleus Test (CSGMT): Sex differences in the micronucleus test. Mutat Res 1986, 172:151-163.

23. Zambrano MA, Targa HJ, Rabello-Gay MN: Physiological saline solutions as a useful tool in micronucleus and metaphase slide preparations. Stain Technol 1982, 57:48-49.
24. Lewi DM, Hopp HE, Escandon AS: Sunflower (Helianthus annuus L.). Methods Mol Biol 2006, 343:291-297.

25. Heo JC, Woo SUK, Kweon MA, Park JY, Lee HK, Son M, Rho JR, Lee SH: Aqueous extract of the Helianthus annuus seed alleviates asthmatic symptoms in vivo. Int J Mol Med Chem 2008, 21:57-61.

26. Cardoso CC, Rodrigues KL, Pichara NL, Dall'Aglio R, Fiorini JE, Fraschini F, Diana GM, Drago L, De Vecchi E, Carvalho JCT: Olio di girasole ozonizzato associate ad acido a-lipoico e a lattobacilli: studio pre-clinico dell'azione antiulcerosa, antinfiammatoria e antibatterica. Farmaci 2004, 28:97-110.

27. Ricardo GA, Zullyt ZR, Yilian L, Hernández F, Menéndez S: Efecto del OLEOZON ${ }^{\circ}$ frente a lesiones gástricas inducidas por indometacina en ratas (Effect of OLEOZON ${ }^{\circ}$ on gastric lesions induced by indomethacin in rats). Revista electrónica de Veterinaria 2007, 8:1-6.

28. Rodrigues KL, Cardoso CC, Caputo LR, Carvalho JC, Fiorini JE, Schneedorf JM: Cicatrizing and antimicrobial properties of an ozonised oil from sunflower seeds. Inflammopharmacology 2004, 3:261-270.

29. Akihisa T, Yasukawa K, Oinuma H, Kasahara Y, Kimura Y, Takase S, Yamanouchi S, Takido M, Kumaki K, Tamura T: Triterpene alcohols from the flowers of Compositae and their anti-inflammatory effects. Phytochemistry 1996, 43:1255-1260.

30. Rojas-Molina M, Campos-Sanches J, Analla M, Serrano M, Moraga AA: Genotoxicity of vegetable cooking oils in the Drosophila wing spot test. Environ Mol Mutagen 2005, 45:90-95.

31. Montero ACR, Carvajal YG, Rodríguez ZZ, López GF, Mirabal JM: Evaluación genotóxica del OLEOZON mediante los ensayos de micronúcleos en medula ósea y sangre periférica de ratón. Revista CENIC: Ciências Biológicas 1998, 29:200-202.

32. Salerno JW, Smith DE: The use of sesame oil and other vegetable oils in the inhibition of human colon cancer growth in vitro. Anticancer Res 1991, 11:209-215.

33. Cognault $\mathrm{S}$, Jourdan ML, Germain E, Pitavy R, Morel E, Durand G, Bougnoux P, Lhuillery C: Effect of an m-linolenic acid-rich diet on rat mammary tumour growth depends on the dietary oxidative status. Nutr Cancer 2000, 36:33-41.

34. Nakayama M, Ju HR, Sugano M, Hirose N, Ueki T, Doi F, Eynard AR: Effect of dietary fat and cholesterol on dimethylbenz [a]-antracene-induced mammary tumorigenesis in Sprague-Dawley rats. Anticancer Res 1993, 13:691-698.

35. La Vecchia C, Negri E, Franceschi S, Decarli A, Giacosa A, Lipworth L: Olive oil, other dietary fats, and the risk of breast cancer (Italy). Cancer Cause Control 1995, 6:545-550.

36. Rao GN, Ney E, Herbert RA: Effect of melatonin and linolenic acid on mammary cancer in transgenic mice with c-neu breast cancer oncogen. Breast Cancer Res 2000, 64:287-296.

37. Burns CP, Luttenegger DG, Specctor AA: Effect of dietary fat saturation on survival of mice with L1210 Leukemia. J Natl Cancer Inst 1978, 61:513-515.

38. Bartsch H, Nai J, Owen RW: Dietary polyunsaturated fatty acids and cancer of the breast and colorectum: emerging evidence for their role as risk modifiers. Carcinogenesis 1999, 20:2209-2218.

39. Boovens J, Engelbrecht P, Le Roux S, Louwrens CC, Van der Merwe CF, Katzeff IE: Some effects of the essential fatty acids linoleic acid and alpha-Linolenic acid and of their metabolites gamma-Linolenic acid, arachidonic acid, eicosapentaenoic acid, docosahesaenoic acid, and of prostaglandins $\mathrm{A} 1$ and $\mathrm{E} 1$ in the proliferation of human osteogenic sarcoma cells in culture. Prostaglandins Leukot Med 1984, 15:15-33.

40. Johanning GL, Lin TZ: Unsaturated fatty acid effects on human breast cancer cell adhesion. Nutr Cancer 1995, 24:57-66.

41. Newcomer LM, King IB, Wicklund KG, Stanford JL: The association of fatty acids with prostate cancer risk. Prostate 2001, 47:262-268.

42. Iwado H, Naito M, Hayatsu H: Genotoxicity and antigenotoxicity of air-borne particulates. Mutat Res 1991, 246:93-103.

43. Siegel I, Liu TL, Yaghoubzadeh E, Keskey TS, Gleicher N: Cytotoxic effects of free fatty acids on ascites tumor cells. J Natl Cancer Inst Monographs 1987, 78:271-277.

44. Bégin LR, Clement PB, Kirk ME, Jothy S, McCaughey WT, Ferenczy A: Aggressive angiomyxoma of pelvic soft parts: a clinicopathologic study of nine cases. Hum Pathol 1985, 16:621-628.

45. Ramon JM, Bou R, Romea S, Alkiza ME, Jacas M, Ribes J, Oromi J: Dietary fat intake and prostate cancer risk: a case-control study in Spain. Cancer Cause Control 2000, 11:679-685. 
46. DeFlora S, Ramel C: Mechanisms of inhibitors of mutagenesis and carcinogenesis: classification and review. Mutat Res 1988, 202:285-306.

47. Visioli F, Bellosta S, Galli C: Oleuropein, the bitter principle of olives, enhances nitric-oxide production by mouse macrophages. Life Sci 1998, 62:541-546.

48. Santos JH, Graf U, Reguly ML, De Andrade HHR: The synergistic effects of vanillin on recombination predominate over its antigenotoxic action in relation to MMC-induced lesions in somatic cells of Drosophila melanogaster. Mutat Res 1999, 444:355-365.

49. Weisburger $\mathrm{JH}$ : Can cancer risks be altered by changing nutritional traditions? Cancer 1998, 83:1278-1281.

50. Katiyar SK, Mohan RR, Agarwal R, Mukhtar H: Protection against induction of mouse skin papillomas with low and high risk of conversion to malignancy by green tea polyphenols. Carcinogenesis 1997, 18:497-502.

51. Indart A, Viana M, Grootveld MC, Silwood CJ, Sanchez-Vera I, Bonet B: Teratogenic actions of thermally-stressed culinary oils in rats. Free Radic Res Commun Commun 2002, 36:1051-1058.

52. Al-Shabanah OA: Inhibition of adriamycin-induced micronuclei by desferrioxamine in Swiss albino mice. Mutat Res 1993, 301:107-111.

53. Bean CL, Armstrong MJ, Galloway SM: Effect of sampling time on chromosome aberration yield for 7 chemicals in Chinese hamster ovary cells. Mutat Res 1992, 265:31-44.

54. Delvaeye M, Verovski V, De Neve W, Storme G: DNA breakage, cytotoxicity, drug accumulation and retention in two human ovarian tumor cell lines AZ224 and AZ364 treated with adriamycin, modulated by verapamil. Anticancer Res 1993, 13:1533-1538.

55. Dhawan A, Kayani MA, Parry JM, Parry E, Anderson D: Aneugenic and clastogenic effects of doxorubicin in human lymphocytes. Mutagenesis 2003, 18:487-490.

56. Jagetia GC, Nayak V: Effect of doxorubicin on cell survival and micronuclei formation in HeLa cells exposed to different doses of gamma-radiation. Strahlenther Onkol 2000, 176:422-428.

57. Vidhya N, Devraj SN: Antioxidant effect of eugenol in rat intestine. Ind J Exp Biol 1999, 37:1192-1195.

doi:10.1186/1472-6882-14-121

Cite this article as: Boriollo et al: Nongenotoxic effects and a reduction of the DXR-induced genotoxic effects of Helianthus annuus Linné (sunflower) seeds revealed by micronucleus assays in mouse bone marrow. BMC Complementary and Alternative Medicine 2014 14:121.

\section{Submit your next manuscript to BioMed Central and take full advantage of:}

- Convenient online submission

- Thorough peer review

- No space constraints or color figure charges

- Immediate publication on acceptance

- Inclusion in PubMed, CAS, Scopus and Google Scholar

- Research which is freely available for redistribution

Submit your manuscript at www.biomedcentral.com/submit
(O) Biomed Central 